\title{
Green kernel estimates and the full Martin boundary for random walks on lamplighter groups and Diestel-Leader graphs
}

\author{
Sara Brofferio ${ }^{1}$, Wolfgang Woess * \\ Institut für Mathematik C, Technische Universität Graz, Steyrergasse 30, 8010 Graz, Austria
}

Received 5 April 2004; accepted 22 December 2004

\begin{abstract}
We determine the precise asymptotic behaviour (in space) of the Green kernel of simple random walk with drift on the Diestel-Leader graph $\mathrm{DL}(q, r)$, where $q, r \geqslant 2$. The latter is the horocyclic product of two homogeneous trees with respective degrees $q+1$ and $r+1$. When $q=r$, it is the Cayley graph of the wreath product (lamplighter group) $\mathbb{Z}_{q} 2 \mathbb{Z}$ with respect to a natural set of generators. We describe the full Martin compactification of these random walks on DL-graphs and, in particular, lamplighter groups. This completes previous results of Woess, who has determined all minimal positive harmonic functions.

(C) 2005 Elsevier SAS. All rights reserved.
\end{abstract}

\section{Résumé}

On détermine le comportement asymptotique précis (dans l'espace) du noyau de Green de la marche aléatoire simple avec dérive sur le graphe de Diestel-Leader $\mathrm{DL}(q, r)$, où $q, r \geqslant 2$. Ce graphe est le produit horocyclique de deux arbres homogènes de degrés $q+1$ et $r+1$, respectivement. Quand $q=r$, il s'agit du graphe de Cayley du produit en couronne («lamplighter group 》) $\mathbb{Z}_{q}$ ZZ par rapport à un ensemble naturel de générateurs. On décrit la compactification de Martin complète de ces marches aléatoires sur les graphes DL, et en particulier, les groupes du «lamplighter». Ceci complète les résultats précédents de Woess, qui a déterminé les fonctions harmoniques minimales.

(c) 2005 Elsevier SAS. All rights reserved.

MSC: 60J50; 05C25; 20E22; 31C05; 60G50

Keywords: Lamplighter group; Wreath product; Diestel-Leader graph; Random walk; Martin boundary; Harmonic functions

\footnotetext{
Supported by European Commission, Marie Curie Fellowship HPMF-CT-2002-02137 and partially by FWF (Austrian Science Fund) project P15577.

* Corresponding author.

E-mail address: woess@weyl.math.tu-graz.ac.at (W. Woess).

1 Current address: Laboratoire de Mathématiques, Université Paris-Sud, bâtiment 425, 91405 Orsay cedex, France.
} 


\section{Introduction}

Consider the additive group $\mathbb{Z}$ of all integers as a two-way-infinite road where at each point there is a lamp that may be switched on in one of $q$ different intensities (states) $\in \mathbb{Z}_{q}=\{0, \ldots, q-1\}$, the group of integers modulo $q$. At the beginning, all lamps are in state 0 (switched off), and a lamplighter starts at some point of $\mathbb{Z}$. He chooses at random among the following actions (or a suitable combination thereof): he can move to a neighbour point in $\mathbb{Z}$, or he can change the intensity of the lamp at the actual site to a different state. As the process evolves, we have to keep track of the position $k \in \mathbb{Z}$ of the lamplighter and of the finitely supported configuration $\eta: \mathbb{Z} \rightarrow \mathbb{Z}_{q}$ that describes the states of all lamps. The set $\mathbb{Z}_{q} 2 \mathbb{Z}$ of all pairs $(\eta, k)$ of this type carries the structure of a semi-direct product of $\mathbb{Z}$ with the additive group $\mathcal{C}$ of all configurations, on which $\mathbb{Z}$ acts by translations. This is often called the lamplighter group; the underlying algebraic construction is the wreath product of two groups.

Random walks on lamplighter groups have been a well-studied subject in recent years, see Kaimanovich and Vershik [18] and Kaimanovich [17] (Poisson boundary $\equiv$ bounded harmonic functions), Lyons, Pemantle and Peres [20], Erschler [12], Revelle [24], Bertacchi [3] (rate of escape), Grigorchuk and Żuk [13], Dicks and Schick [7], Bartholdi and Woess [2] (spectral theory), Saloff-Coste and Pittet [22,23], Revelle [25] (asymptotic behaviour of transition probabilities), and Woess [28] (positive harmonic functions).

Here, we shall deal with Green kernel asymptotics and positive harmonic functions. Let us briefly outline in general how this is linked with Martin boundary theory of Markov chains. Consider an arbitrary infinite (connected, locally finite) graph $X$ (e.g., a Cayley graph of a finitely generated group) and the stochastic transition matrix $P=(p(x, y))_{x, y \in X}$ of a random walk $Z_{n}$ on $X$. That is, $Z_{n}$ is an $X$-valued random variable, the position of the random walker at time $n$, subject to the Markovian transition rule

$$
\operatorname{Pr}\left[Z_{n+1}=y \mid Z_{n}=x\right]=p(x, y) .
$$

The $n$-step transition probability

$$
p^{(n)}(x, y)=\operatorname{Pr}\left[Z_{n}=y \mid Z_{0}=x\right], \quad x, y \in X,
$$

is the $(x, y)$-entry of the matrix power $P^{n}$, with $P^{0}=I$, the identity matrix. The Green kernel is

$$
G(x, y)=\sum_{n=0}^{\infty} p^{(n)}(x, y), \quad x, y \in X .
$$

This is the expected number of visits in the point $y$, when the random walk starts at $x$. We always consider random walks that are irreducible and transient, which amounts to

$$
0<G(x, y)<\infty \text { for all } x, y \in X \text {. }
$$

Renewal theory in a wide sense consists in describing the asymptotic behaviour in space of $G(x, y)$, when $x$ is fixed and $y$ tends to infinity (or dually, $y$ is fixed and $x$ tends to infinity). If we fix a reference point $o \in X$, then the Martin kernel is

$$
K(x, y)=G(x, y) / G(o, y), \quad x, y \in X .
$$

If we have precise asymptotic estimates in space of the Green kernel, then we can also determine the Martin compactification. This is the smallest metrizable compactification of $X$ containing $X$ as a discrete, dense subset, and to which all functions $K(x, \cdot), x \in X$, extend continuously. The Martin boundary $\mathcal{M}=\mathcal{M}(P)$ is the ideal boundary added to $X$ in this compactification. Thus, $\mathcal{M}$ consists of the "directions of convergence" of $K(x, y)$, when $y \rightarrow \infty$. Its significance is that it leads to a complete understanding of the cone $\mathcal{H}^{+}=\mathcal{H}^{+}(P)$ of positive harmonic functions. A function $h: X \rightarrow \mathbb{R}$ is called harmonic, or $P$-harmonic, if

$$
h=P h, \quad \text { where } P h(x)=\sum_{y} p(x, y) h(y) .
$$


A function $h \in \mathcal{H}^{+}$is called minimal if

$$
h(o)=1 \quad \text { and } \quad h \geqslant h_{1} \in \mathcal{H}^{+} \Longrightarrow h_{1} / h \equiv \text { constant. }
$$

The minimal harmonic functions are the extreme points of the convex base $\mathcal{B}=\left\{h \in \mathcal{H}^{+}: h(o)=1\right\}$ of the cone $\mathcal{H}^{+}$.

The reader is referred to the excellent introduction to Martin boundary theory by Dynkin [10], based on the pioneering work by Doob [9] and Hunt [16]. A main result of this theory is that

- Every minimal harmonic function is of the form $K(\cdot, \xi)$, where $\xi \in \mathcal{M}$, and the set $\mathcal{M}_{\min }=\{\xi \in \mathcal{M}$ : $K(\cdot, \xi)$ is minimal $\}$ is a Borel subset of $\mathcal{M}$;

- For every $h \in \mathcal{H}^{+}$there is a unique Borel measure $v^{h}$ on $\mathcal{M}$ such that

$$
\nu^{h}\left(\mathcal{M} \backslash \mathcal{M}_{\min }\right)=0 \quad \text { and } \quad h(\cdot)=\int_{\mathcal{M}} K(\cdot, \xi) \mathrm{d} \nu^{h}(\xi) .
$$

The above is an abstract construction of the Martin compactification. The kind of approach that we have in mind here is the following. The transition matrix $P$ is adapted to the graph structure, and we want to understand and describe the Martin compactification in terms of the specific geometry of $X$. Results of this type for random walks on various classes of graphs and groups, along with many references, are presented in the book by Woess [27].

Returning to lamplighter walks, this spirit requires as the starting point a good understanding of the geometry of the wreath product $\mathbb{Z}_{q} 2 \mathbb{Z}$ in terms of a suitable Cayley graph of that group. This is the Diestel-Leader graph $\operatorname{DL}(q, q)$, a special case of the Diestel-Leader graphs $\operatorname{DL}(q, r)(q, r \geqslant 2)$, which were introduced in [8] and are explained in detail in Section 2. Briefly speaking, $\operatorname{DL}(q, r)$ is the horocyclic product of the homogeneous trees $\mathbb{T}_{q}$ and $\mathbb{T}_{r}$ with degrees $q+1$ and $r+1$, respectively. It is precisely this geometric realization of the lamplighter groups in terms of relatively simple objects such as trees, that allows us to perform many computations.

The random walk with transition matrix $P_{\alpha}$ on $\mathrm{DL}(q, r)$ that we consider is the simple random walk (SRW) with an additional drift parameter $\alpha \in(0,1)$. If $r=q$ (the case of the lamplighter group), then this random walk can be interpreted in lamplighter terms as follows. Think of the lamps not placed at each vertex of the two-way-infinite path $\mathbb{Z}$, but at the middle of each edge. Suppose the actual position of the lamplighter is $k \in \mathbb{Z}$. He first tosses a coin. If "head" comes up, which happens with probability $\alpha$, he moves to $k+1$ and switches the lamp on the transversed edge to a state chosen at random in $\mathbb{Z}_{q}$. Otherwise, he moves to $k-1$ and also switches the lamp on the transversed edge to a random state.

Even when $q \neq r$, the random walk $P_{\alpha}$ on $\mathrm{DL}(q, r)$ may be interpreted as a lamplighter walk in an extended sense. Imagine that on each edge of $\mathbb{Z}$, there is a green lamp with $q$ possible intensities (including "off") in $\mathbb{Z}_{q}$ plus a red lamp with $r$ possible intensities (including "off”) in $\mathbb{Z}_{r}$. The rule is that only finitely many lamps may be switched on, and in addition, if the lamplighter stands at $k$, then all red lamps between $k$ and $-\infty$ have to be switched off, while all green lamps between $k$ and $+\infty$ must be switched off. The lamplighter tosses his $\alpha$-coin. If "head" comes up, he moves from $k$ to $k+1$ and switches the green lamp on the transversed edge to a random state, while switching off the red lamp on that edge. Otherwise, he moves to $k-1$ and switches the red lamp on the transversed edge to a random state, while switching off the green lamp sitting there.

Then the random walk $P=P_{\alpha}$ (whose definition is formalised in (2.3)) is irreducible and transient. Via our geometric interpretation, we see that it has natural projections $P_{1}=P_{\alpha, q}$ and $P_{2}=P_{1-\alpha, r}$ on the two trees used to make up the graph, and also $\widetilde{P}=\widetilde{P}_{\alpha}$ on $\mathbb{Z}$, which describes just the moves of the lamplighter. A good understanding of these projected walks is crucial for our approach, and in Section 3, we quickly review the necessary facts concerning those random walks on $\mathbb{T}_{q}$ (and $\mathbb{T}_{r}$ ).

In Section 4, we derive our main results concerning the asymptotic behaviour of the Green kernel associated with $P_{\alpha}$, subsumed in Theorem 4.2. The asymptotics are different along different directions of moving to infinity. Also, the drift-free case $(\alpha=1 / 2)$ is substantially different from the other cases $(\alpha \neq 1 / 2)$. 
These results are used in Section 5 to describe the full Martin compactification. In the drift-free case, this is the "natural" geometric compactification in terms of the two underlying trees. Namely, $\mathrm{DL}(q, r)$ is a subgraph of $\mathbb{T}_{q} \times \mathbb{T}_{r}$, and the Martin compactification is the closure of $\operatorname{DL}(q, r)$ in $\widehat{\mathbb{T}}_{q} \times \widehat{\mathbb{T}}_{r}$, where $\widehat{\mathbb{T}}_{q}$ and $\widehat{\mathbb{T}}_{r}$ are the wellknown end-compactifications of the respective trees. However, when $\alpha \neq 1 / 2$, the Martin compactification is larger than (三 surjects non-trivially onto) the geometric one. The minimal Martin boundary, previously described in [28] without elaborating the directions of convergence, is recovered.

These results can also be adapted to obtain the Martin compactification for all positive $t$-harmonic functions, that satisfy $P h=t \cdot h$, where $t \geqslant \rho\left(P_{\alpha}\right)=\limsup _{n} p^{(n)}(x, x)^{1 / n}$, the "bottom of the positive spectrum". The picture at the bottom is that of the drift-free case, while the case $t>\rho\left(P_{\alpha}\right)$ corresponds to non-vanishing drift. See Section 6.

In the short Section 7, we present another little by-product of our Green kernel estimates, namely, we illustrate their use for showing directly that SRW on $\operatorname{DL}(q, q)$ (the lamplighter group) does not satisfy the elliptic Harnack inequality.

In conclusion, let us remark that in general it is significantly harder to determine the whole Martin compactification than to determine the minimal harmonic functions associated with a random walk, since the former contains more detailed analytic-geometric informations than the latter, whose computation often has rather an algebraic than an analytic flavour. Let us also remark that our results provide the first case where one can successfully determine the whole Martin compactification of a class of random walks on finitely generated groups that are solvable, but do not have polynomial growth.

\section{The geometry of Diestel-Leader graphs and lamplighter groups}

We now explain the structure of the DL-graphs and their relation with the wreath products $\mathbb{Z}_{q} 2 \mathbb{Z}$. This section is a short and slightly modified version of $\$ 2$ in [28], included here for the sake of completeness.

Let $\mathbb{T}=\mathbb{T}_{q}$ be the homogeneous tree with degree $q+1, q \geqslant 2$. A geodesic path, respectively geodesic ray, respectively infinite geodesic in $\mathbb{T}$ is a finite, respectively one-sided infinite, respectively doubly infinite sequence $\left(x_{n}\right)$ of vertices of $\mathbb{T}$ such that $d\left(x_{i}, x_{j}\right)=|i-j|$ for all $i, j$, where $d(\cdot, \cdot)$ denotes the graph distance.

Two rays are equivalent if their symmetric difference is finite. An end of $\mathbb{T}$ is an equivalence class of rays. The space of ends is denoted $\partial \mathbb{T}$, and we write $\widehat{\mathbb{T}}=\mathbb{T} \cup \partial \mathbb{T}$. For all $w, z \in \widehat{\mathbb{T}}, w \neq z$, there is a unique geodesic $\overline{w z}$ that connects the two. In particular, if $x \in \mathbb{T}$ and $\xi \in \partial \mathbb{T}$ then $\overline{x \xi}$ is the ray that starts at $x$ and represents $\xi$.

For $x, y \in \mathbb{T}, x \neq y$, we define the cone $\widehat{\mathbb{T}}(x, y)=\{w \in \widehat{\mathbb{T}}: y \in \overline{x w}\}$. The collection of all cones is a sub-basis of a topology which makes $\widehat{\mathbb{T}}$ a compact, totally disconnected Hausdorff space with $\mathbb{T}$ as a dense, discrete subset.

We fix a root vertex $o \in \mathbb{T}$. If $w, z \in \widehat{\mathbb{T}}$, then their confluent $c=w \wedge z$ with respect to $o$ is defined by $\overline{o w} \cap \overline{o z}=$ $\overline{o c}$. Similarly, we choose and fix a reference end $\omega \in \partial \mathbb{T}$. For $z, v \in \widehat{\mathbb{T}} \backslash\{\omega\}$, their confluent $b=v \curlywedge z$ with respect to $\omega$ is defined by $\overline{v \omega} \cap \overline{z \omega}=\overline{b \omega}$. We write

$$
z \preccurlyeq v \quad \text { if } z \curlywedge v=z \text {. }
$$

For $x, y \in \mathbb{T}$, we describe their relative position by the two numbers

$$
\mathfrak{u}(x, y)=d(x, x \curlywedge y) \quad \text { and } \quad \mathfrak{d}(x, y)=d(y, x \curlywedge y) .
$$

Thus, $\mathfrak{d}(x, y)=\mathfrak{u}(y, x)$. In Fig. $1, \mathfrak{u}(x, y)$ and $\mathfrak{d}(x, y)$ correspond to the numbers of steps one has to take upwards (in direction of $\omega$ ), respectively downwards, on the geodesic path from $x$ to $y$. We have $d(x, y)=\mathfrak{u}(x, y)+\mathfrak{d}(x, y)$.

The Busemann function $\mathfrak{h}: \mathbb{T} \rightarrow \mathbb{Z}$ and the horocycles $H_{k}$ with respect to $\omega$ are

$$
\mathfrak{h}(x)=\mathfrak{d}(o, x)-\mathfrak{u}(o, x) \quad \text { and } \quad H_{k}=\{x \in \mathbb{T}: \mathfrak{h}(x)=k\} .
$$

Every horocycle is infinite. We write $H(x)=H_{k}$ if $x \in H_{k}$. Every vertex $x$ in $H_{k}$ has one neighbour $x^{-}$(its predecessor) in $H_{k-1}$ and $q$ neighbours (its successors) in $H_{k+1}$. Thus $\preccurlyeq$ is the transitive closure of the predecessor relation. We set $\partial^{*} \mathbb{T}=\partial \mathbb{T} \backslash\{\omega\}$. 


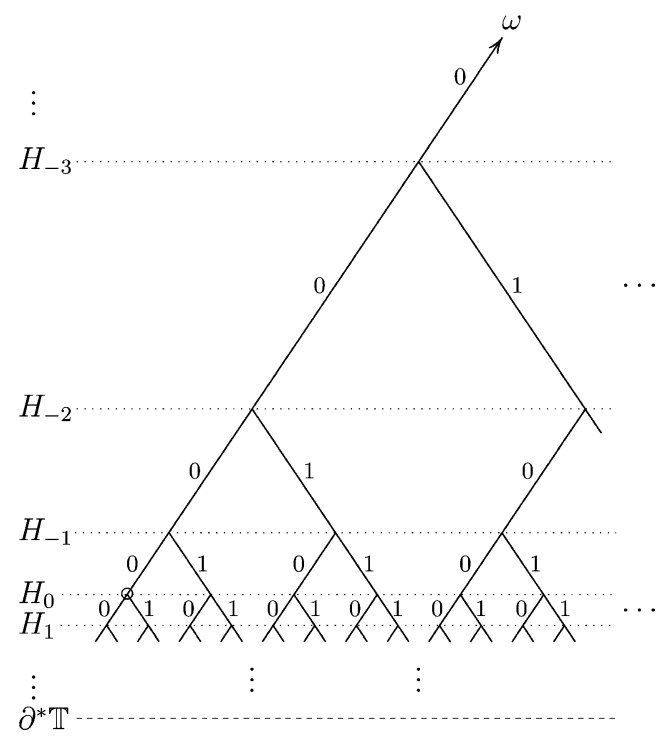

Fig. 1.

We label each edge of $\mathbb{T}$ by an element of $\mathbb{Z}_{q}$ such that for each vertex, the "downward" edges to its $q$ successors carry labels $0, \ldots, q-1$ from left to right (say), see Fig. 1 . Thus, for each $x \in \mathbb{T}$, the sequence $(\sigma(n))_{n \leqslant 0}$ of labels on the geodesic $\overline{x \omega}$ has finite support $\{n: \sigma(n) \neq 0\}$. We write $\Sigma_{q}$ for the set of all those sequences. On every horocycle, there is exactly one vertex corresponding to each $\sigma \in \Sigma_{q}$. Thus, $\mathbb{T}_{q}$ is in one-to-one correspondence with the set $\Sigma_{q} \times \mathbb{Z}$, and the $k$-th horocycle is $H_{k}=\Sigma_{q} \times\{k\}$.

Now consider two trees $\mathbb{T}^{1}=\mathbb{T}_{q}$ and $\mathbb{T}^{2}=\mathbb{T}_{r}$ with roots $o_{1}$ and $o_{2}$ and reference ends $\omega_{1}$ and $\omega_{2}$, respectively.

2.1. Definition. The Diestel-Leader graph $\mathrm{DL}(q, r)$ is

$$
\operatorname{DL}(q, r)=\left\{x_{1} x_{2} \in \mathbb{T}_{q} \times \mathbb{T}_{r}: \mathfrak{h}\left(x_{1}\right)+\mathfrak{h}\left(x_{2}\right)=0\right\},
$$

and neighbourhood is given by

$$
x_{1} x_{2} \sim y_{1} y_{2} \Longleftrightarrow x_{1} \sim y_{1} \quad \text { and } \quad x_{2} \sim y_{2} .
$$

To visualise $\operatorname{DL}(q, r)$, draw $\mathbb{T}_{q}$ in horocyclic layers with $\omega_{1}$ at the top and $\partial^{*} \mathbb{T}_{q}$ at the bottom, and right to it $\mathbb{T}_{r}$ in the same way, but upside down, with the respective horocycles $H_{k}\left(\mathbb{T}_{q}\right)$ and $H_{-k}\left(\mathbb{T}_{r}\right)$ aligned. Connect the two origins $o_{1}, o_{2}$ by an elastic spring. It is allowed to move along each of the two trees, may expand infinitely, but must always remain in horizontal position. The vertex set of $\mathrm{DL}_{q, r}$ consists of all admissible positions of the spring. From a position $x_{1} x_{2}$ with $\mathfrak{h}\left(x_{1}\right)+\mathfrak{h}\left(x_{2}\right)=0$ the spring may move upwards to one of the $r$ successors of $x_{2}$ in $\mathbb{T}_{r}$, and at the same time to the predecessor of $x_{1}$ in $\mathbb{T}_{q}$, or it may move downwards in the analogous way. Such a move corresponds to going to a neighbour of $x_{1} x_{2}$. We see that $\operatorname{DL}(q, r)$ is regular with degree $q+r$. As the reference point in $\operatorname{DL}(q, r)$, we choose $o=o_{1} o_{2}$. Fig. 2 illustrates $\operatorname{DL}(2,2)$.

The position of $y=y_{1} y_{2}$ with respect to $x_{1} x_{2} \in \operatorname{DL}(q, r)$ is described by the four numbers $\mathfrak{u}\left(x_{1}, y_{1}\right), \mathfrak{d}\left(x_{1}, y_{1}\right)$, $\mathfrak{u}\left(x_{2}, y_{2}\right), \mathfrak{d}\left(x_{2}, y_{2}\right)$, see below in Section 4, (4.1) and Fig. 3. The random walks that we are going to deal with are all such that the transition probabilities $p\left(x_{1} x_{2}, y_{2} y_{2}\right)$ depend only on those four parameters - a crucial prerequisite for our approach.

We now recall in more detail the construction of the lamplighter group $\mathbb{Z}_{q} 2 \mathbb{Z}$. The group of all finitely supported configurations is

$$
\mathcal{C}=\left\{\eta: \mathbb{Z} \rightarrow \mathbb{Z}_{q}, \operatorname{supp}(\eta) \text { finite }\right\}
$$




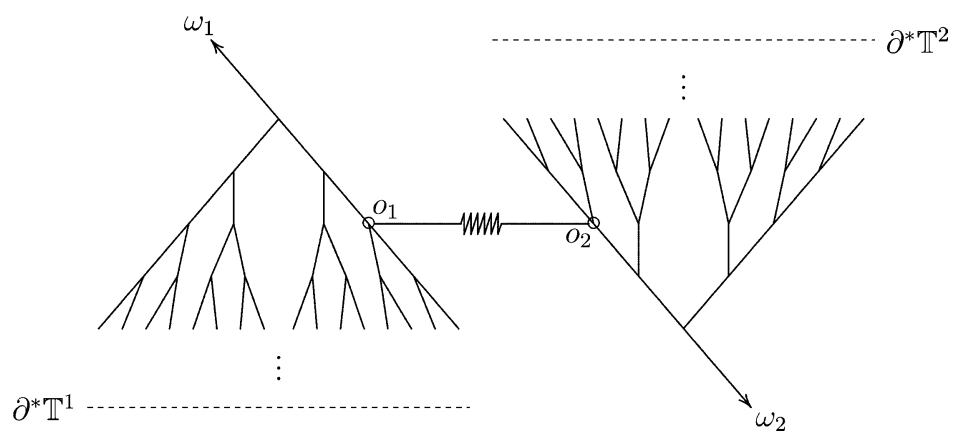

Fig. 2.

with point-wise addition modulo $q$. The group $\mathbb{Z}$ acts on $\mathcal{C}$ by translations $k \mapsto T_{k}: \mathcal{C} \rightarrow \mathcal{C}$ with $T_{k} \eta(m)=\eta(m-k)$. The resulting semi-direct product $\mathbb{Z} \wedge \mathcal{C}$ is

$$
\mathbb{Z}_{q} 2 \mathbb{Z}=\{(\eta, k): \eta \in \mathcal{C}, k \in \mathbb{Z}\} \quad \text { with group operation } \quad(\eta, k)\left(\eta^{\prime}, k^{\prime}\right)=\left(\eta+T_{k} \eta^{\prime}, k+k^{\prime}\right) .
$$

We identify each $(\eta, k) \in \mathbb{Z}_{q} 2 \mathbb{Z}$ with the vertex $x_{1} x_{2} \in \mathrm{DL}(q, q)$, where according to the identification $\mathbb{T}_{q} \leftrightarrow$ $\Sigma_{q} \times \mathbb{Z}$, the vertices $x_{i}$ are given by

$$
\begin{aligned}
& x_{1}=\left(\eta_{k}^{-}, k\right) \text { and } x_{2}=\left(\eta_{k}^{+},-k\right), \text { where } \\
& \eta_{k}^{-}=\left.\eta\right|_{(-\infty, k]} \text { and } \eta_{k}^{+}=\left.\eta\right|_{[k+1, \infty)},
\end{aligned}
$$

both written as sequences over the non-positive integers.

This is a one-to-one correspondence between $\mathbb{Z}_{q} 2 \mathbb{Z}$ and $\mathrm{DL}(q, q)$, and that group acts transitively and fixedpoint-freely on the graph. Namely, the action of $m \in \mathbb{Z}$ is given by $x_{1} x_{2}=\left(\sigma_{1}, k\right)\left(\sigma_{2},-k\right) \mapsto y_{1} y_{2}=\left(\sigma_{1}, k+\right.$ $m)\left(\sigma_{2},-k-m\right)$, and the action of the group of configurations is point-wise addition modulo $q$. Write $\delta_{k}^{l}$ for the configuration in $\mathcal{C}$ with value $l$ at $k$ and 0 elsewhere. Then $\operatorname{DL}(q, q)$ is the (right) Cayley graph of $\mathbb{Z}_{q} \imath \mathbb{Z}$ with respect to the symmetric set of generators

$$
\left\{\left(\delta_{1}^{l}, 1\right),\left(\delta_{0}^{l},-1\right): l \in \mathbb{Z}_{q}\right\},
$$

i.e., an edge corresponds to multiplying with a generator on the right. (This is precisely the set of generators considered in [13] and [7] when computing the spectrum of the associated SRW-operator.)

Returning to $\mathrm{DL}=\mathrm{DL}(q, r)$, the transition matrix $P_{\alpha}$ of the random walk that we have described in the Introduction is given as follows. For $x=x_{1} x_{2}, y=y_{1} y_{2} \in \mathrm{DL}(q, r)$

$$
p_{\alpha}(x, y)= \begin{cases}\alpha / q & \text { if } y_{1}^{-}=x_{1} \text { and } y_{2}=x_{2}^{-}, \\ (1-\alpha) / r & \text { if } y_{1}=x_{1}^{-} \text {and } y_{2}^{-}=x_{2} \\ 0 & \text { otherwise. }\end{cases}
$$

\section{Simple random walk with drift on a homogeneous tree}

In general, if $P$ is a transition matrix over a set $X$ and $\left\{X_{i}: i \in I\right\}$ is a partition of $X$ with the associated quotient map $\pi: X \rightarrow I$, then one says that $P$ factorises (or projects) with respect to $\pi$, if $\tilde{p}(i, j):=\sum_{y \in X_{j}} p(x, y)$ does not depend on the specific choice of $x \in X_{i}$. In this case, the Green kernel $\widetilde{G}$ associated with $\widetilde{P}=\pi(P)$ also satisfies

$$
\widetilde{G}(i, j)=\sum_{y \in X_{j}} G(x, y), \quad x \in X_{i} .
$$


In our case, we have three natural, neighbourhood preserving projections $\pi_{1}: \mathrm{DL} \rightarrow \mathbb{T}_{q}, \pi_{2}: \mathrm{DL} \rightarrow \mathbb{T}_{r}$, and $\tilde{\pi}: \mathrm{DL} \rightarrow \mathbb{Z}$, given by

$$
\pi_{1}\left(x_{1} x_{2}\right)=x_{1}, \quad \pi_{2}\left(x_{1} x_{2}\right)=x_{2}, \quad \text { and } \quad \tilde{\pi}\left(x_{1} x_{2}\right)=\mathfrak{h}\left(x_{1}\right) .
$$

$P_{\alpha}$ factorises with respect to each of them. Let $\pi_{1}\left(P_{\alpha}\right)=P_{1}, \pi_{2}\left(P_{\alpha}\right)=P_{2}$ and $\tilde{\pi}\left(P_{\alpha}\right)=\widetilde{P}$. Then $P_{1}=P_{\alpha, q}$ on $\mathbb{T}^{1}=\mathbb{T}_{q}, P_{2}=P_{1-\alpha, r}$ on $\mathbb{T}^{2}=\mathbb{T}_{r}$, and $\widetilde{P}=\widetilde{P}_{\alpha}$ on $\mathbb{Z}$, where

$$
p_{\alpha, q}\left(x_{1}, y_{1}\right)=\left\{\begin{array}{ll}
\alpha / q & \text { if } y_{1}^{-}=x_{1}, \\
1-\alpha & \text { if } y_{1}=x_{1}^{-}, \\
0 & \text { otherwise, }
\end{array} \quad \tilde{p}_{\alpha}(k, l)= \begin{cases}\alpha & \text { if } l=k+1, \\
1-\alpha & \text { if } l=k-1, \\
0 & \text { otherwise }\end{cases}\right.
$$

The projected random walks are well understood. Everybody is familiar with the gambler's process $\widetilde{P}_{\alpha}$ on $\mathbb{Z}$. We outline the basic features of $P_{\alpha, q}$ on $\mathbb{T}_{q}$ (or, equivalently, $P_{1-\alpha, r}$ on $\mathbb{T}_{r}$ ).

Spectral radius. The spectral radius of any irreducible transition matrix is defined as

$$
\rho(P)=\limsup _{n} p^{(n)}(x, y)^{1 / n} .
$$

It is independent of $x, y$. In the specific case of our random walks with drift parameter $\alpha$, we have

$$
\rho\left(P_{\alpha}\right)_{\mathrm{DL}}=\rho\left(P_{\alpha, q}\right)_{\mathbb{T}_{q}}=\rho\left(P_{1-\alpha, r}\right)_{\mathbb{T}_{r}}=\rho\left(\widetilde{P}_{\alpha}\right)_{\mathbb{Z}}=2 \sqrt{\alpha(1-\alpha)} .
$$

(The subscript refers to the respective underlying graph.) For $\widetilde{P}_{\alpha}$ on $\mathbb{Z}$, this is well known. For $P_{\alpha, q}$ on $\mathbb{T}_{q}$, it can be easily computed in various ways. See e.g. Saloff-Coste and Woess [26], Example 1.

Green kernel. The - simple - computations of the Green kernel $G_{1}=G_{\alpha, q}$ associated with $P_{\alpha, q}$ can be done following the method of §1.D in [27], see also [28], (3.9). The main point is that we have a nearest neighbour random walk on a tree (transition probabilities are positive only between neighbours). Thus, if $F_{1}\left(x_{1}, y_{1}\right)$ is the probability that the random walk starting at $x_{1}$ ever hits $y_{1}\left(x_{1}, y_{1} \in \mathbb{T}_{q}\right)$, then

$$
F_{1}\left(x_{1}, y_{1}\right)=F_{1}\left(x_{1}, w_{1}\right) F_{1}\left(w_{1}, y_{1}\right) \text { for all } w_{1} \in \overline{x_{1} y_{1}} .
$$

Furthermore, since $p_{1}\left(x_{1}, y_{1}\right)$ depends only on $\mathfrak{u}\left(x_{1}, y_{1}\right)$ and $\mathfrak{d}\left(x_{1}, y_{1}\right)$, the same is true for $F_{1}\left(x_{1}, y_{1}\right)$ and $G_{1}\left(x_{1}, y_{1}\right)$. In particular,

$$
F_{1}^{-}=F_{1}\left(x_{1}, x_{1}^{-}\right) \quad \text { and } \quad F_{1}^{+}=F_{1}\left(x_{1}^{-}, x_{1}\right)
$$

are independent of $x_{1} \in \mathbb{T}_{q}$ as well as $G_{1}\left(x_{1}, x_{1}\right)$. Using these facts, and setting $\alpha^{+}=\max \{\alpha, 1-\alpha\}$, one computes

$$
G_{1}\left(x_{1}, y_{1}\right)=F_{1}\left(x_{1}, y_{1}\right) G_{1}\left(y_{1}, y_{1}\right)=\left(F_{1}^{-}\right)^{\mathfrak{u}\left(x_{1}, y_{1}\right)}\left(F_{1}^{+}\right)^{\mathfrak{d}\left(x_{1}, y_{1}\right)} \frac{q}{(q+1) \alpha^{+}-1},
$$

where

$$
F_{1}^{-}=\left\{\begin{array}{ll}
\frac{1-\alpha}{\alpha} & \text { if } \alpha \geqslant \frac{1}{2}, \\
1 & \text { if } \alpha \leqslant \frac{1}{2},
\end{array} \quad F_{1}^{+}= \begin{cases}\frac{1}{q} & \text { if } \alpha \geqslant \frac{1}{2}, \\
\frac{\alpha}{(1-\alpha) q} & \text { if } \alpha \leqslant \frac{1}{2} .\end{cases}\right.
$$

Martin compactification. By (3.4), the Martin kernel $K_{1}=K_{\alpha, q}$ associated with $P_{\alpha, q}$ satisfies

$$
K_{1}\left(x_{1}, y_{1}\right)=\frac{F_{1}\left(x_{1}, y_{1}\right)}{F_{1}\left(o_{1}, y_{1}\right)}=\frac{F_{1}\left(x_{1}, c_{1}\right)}{F_{1}\left(o_{1}, c_{1}\right)}, \quad \text { where } c_{1}=x_{1} \wedge y_{1}
$$

(the confluent with respect to $o_{1}$ ). From here, the following is almost immediate. 
3.6. Proposition. The Martin compactification of $\mathbb{T}_{q}$ with respect to $P_{\alpha, q}$ is the end compactification $\widehat{\mathbb{T}}_{q}$, and for $\xi_{1} \in \partial \mathbb{T}_{q}$, the Martin kernel is given by $K_{1}\left(x_{1}, \xi_{1}\right)=K_{1}\left(x_{1}, c_{1}\right)$, where $c_{1}=x_{1} \wedge \xi_{1}$.

Furthermore, each function $K_{1}\left(\cdot, \xi_{1}\right), \xi_{1} \in \partial \mathbb{T}_{q}$, is minimal harmonic for $P_{\alpha, q}$.

For general transient nearest neighbour random walks on arbitrary locally finite trees, this is due to by Cartier [4], and in the specific case of free groups (which is close to, but not identical with our situation), it was shown previously by Dynkin and Malyutov [11].

The analogous results for $P_{1-\alpha, r}$ on $\mathbb{T}_{r}$ are obtained from the above by exchanging $\alpha$ with $1-\alpha$ and $q$ with $r$. When $\alpha \neq 1 / 2$, the same computations are also valid for $\widetilde{P}_{\alpha}$ on $\mathbb{Z}$, setting $q=1$. When $\alpha=1 / 2$ then $\widetilde{P}_{\alpha}$ is of course recurrent, i.e., the associated Green kernel diverges.

Below in Section 5, we shall also need the following functions on $\mathbb{T}_{q} \times \mathbb{T}_{q}$, which we call (generalised) spherical functions. We set $\mathfrak{u}=\mathfrak{u}\left(x_{1}, y_{1}\right), \mathfrak{d}=\mathfrak{d}\left(x_{1}, y_{1}\right)$ and $\beta=(1-\alpha) / \alpha$.

$$
\phi_{\alpha, q}\left(x_{1}, y_{1}\right)= \begin{cases}\frac{1}{q^{\mathfrak{d}}}\left(\frac{q+1}{q-1}+d\left(x_{1}, y_{1}\right)\right), & \text { if } \alpha=\frac{1}{2}, \\ \frac{1}{\left(q \beta^{2}\right)^{\mathfrak{d}}}\left(\frac{q \beta+1}{q \beta^{2}-1}+\frac{\beta^{\mathfrak{u}}-1}{\beta-1}+\frac{\beta^{\mathfrak{d}}-1}{\beta-1}\right) & \text { if } \alpha<\frac{1}{2}, \\ \frac{\beta^{d\left(x_{1}, y_{1}\right)}}{q^{\mathfrak{d}}}\left(\frac{q \beta^{-1}+1}{q \beta^{-2}-1}+\frac{\beta^{-\mathfrak{u}}-1}{\beta^{-1}-1}+\frac{\beta^{-\mathfrak{d}}-1}{\beta^{-1}-1}\right) & \text { if } \alpha>\frac{1}{2} .\end{cases}
$$

(Recall that $d\left(x_{1}, y_{1}\right)=\mathfrak{u}+\mathfrak{d}$.) Then $\phi_{\alpha, q}\left(\cdot, y_{1}\right)$ is $P_{\alpha, q}$-harmonic on $\mathbb{T}_{q}$ for each $y_{1} \in \mathbb{T}_{q}$.

\section{Green kernel asymptotics}

We now embark on the main computational part of this paper. We consider $P_{\alpha}$ on $\mathrm{DL}=\mathrm{DL}(q, r)$, and we shall always assume that $\alpha \leqslant 1 / 2$, since all results in the case $\alpha \geqslant 1 / 2$ are obtained from the former by exchanging the role of the two trees (i.e., exchanging $r$ with $q$ ).

We want to derive asymptotic estimates of the associated Green kernel $G(x, y)=G_{\alpha}(x, y)$, where $x=x_{1} x_{2}$ and $y=y_{1} y_{2} \in D L$ and the graph distance $d(x, y) \rightarrow \infty$. The latter means that at least one of $d\left(x_{1}, y_{1}\right)$ and $d\left(x_{2}, y_{2}\right)$ (distances in the respective trees) tends to $\infty$. We remark here that

$$
\begin{aligned}
& d(x, y)=d\left(x_{1}, y_{1}\right)+d\left(x_{2}, y_{2}\right)-\left|\mathfrak{h}\left(y_{1}\right)-\mathfrak{h}\left(x_{1}\right)\right|, \\
& d\left(x_{i}, y_{i}\right)=\mathfrak{u}_{i}+\mathfrak{d}_{i}, \quad \mathfrak{h}\left(y_{i}\right)-\mathfrak{h}\left(x_{i}\right)=\mathfrak{d}_{i}-\mathfrak{u}_{i}, \quad(i=1,2), \quad \text { and } \\
& \mathfrak{u}_{1}+\mathfrak{u}_{2}=\mathfrak{d}_{1}+\mathfrak{d}_{2}, \quad \text { where } \mathfrak{u}_{i}=\mathfrak{u}\left(x_{i}, y_{i}\right) \text { and } \mathfrak{d}_{i}=\mathfrak{d}\left(x_{i}, y_{i}\right) .
\end{aligned}
$$

(Cf. Bertacchi [3] for the distance formula.) In terms of the lamplighter moving along $\mathbb{Z}$ (with the lamps - possibly red and green - sitting on the edges, as described in the Introduction), $\mathfrak{u}_{1}$ is the minimal number of steps the lamplighter has to walk in the negative direction in order to obtain the new position and configuration encoded in the vertex $y=y_{1} y_{2}$ of $\mathrm{DL}$, and $\mathfrak{u}_{2}$ is analogous in the positive direction.

We set $c_{i}=x_{i} \curlywedge y_{i}$. See Fig. 3. We also choose $a_{i}, b_{i} \in \mathbb{T}^{i}$ with $x_{i} \preccurlyeq a_{i}, y_{i} \preccurlyeq b_{i}$, such that $\mathfrak{h}\left(a_{1}\right)=\mathfrak{h}\left(b_{1}\right)=$ $-\mathfrak{h}\left(c_{2}\right)$ and $\mathfrak{h}\left(a_{2}\right)=\mathfrak{h}\left(b_{2}\right)=-\mathfrak{h}\left(c_{1}\right)$, i.e., the pairs $a_{1} c_{2}, b_{1} c_{2}, c_{1} a_{2}, c_{1} b_{2}$ belong to DL. In particular, $d(x, y) \rightarrow \infty$ means that $\mathfrak{s} \rightarrow \infty$, where

$$
\mathfrak{s}=\mathfrak{s}(x, y)=\mathfrak{u}_{1}+\mathfrak{u}_{2}=\mathfrak{d}_{1}+\mathfrak{d}_{2}=-\mathfrak{h}\left(c_{1}\right)-\mathfrak{h}\left(c_{2}\right)
$$

is the span of $x$ and $y$.

The following is the first main result of this paper. 


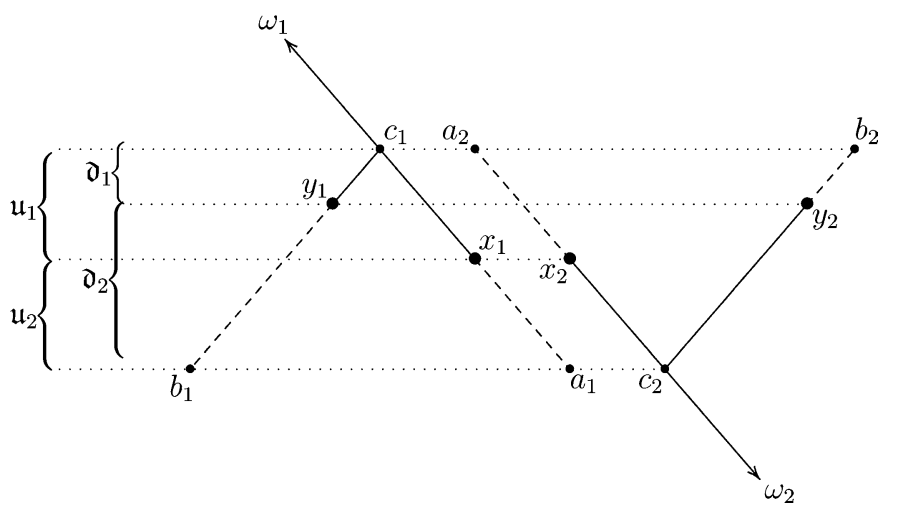

Fig. 3.

4.2. Theorem. Referring to (4.1) and Fig. 3, suppose that $d(x, y) \rightarrow \infty$, and hence $\mathfrak{s}=\mathfrak{s}(x, y) \rightarrow \infty$. Then we have the following.

(a) If $\alpha<1 / 2$ and $\beta=(1-\alpha) / \alpha$ then

$$
\begin{aligned}
& G(x, y) \sim \frac{A_{\beta}}{(q \beta)^{\mathfrak{d}_{1}} r^{\mathfrak{d}_{2}}}(\left(B \beta \frac{\beta^{\mathfrak{s}}-\beta^{\mathfrak{u}_{1}}}{\beta^{\mathfrak{s}}-1} \frac{\beta^{\mathfrak{s}}-\beta^{\mathfrak{d}_{1}}}{\beta^{\mathfrak{s}}-1} \frac{1}{\beta^{\mathfrak{s}}}+\frac{\beta^{\mathfrak{s}}-\beta^{\mathfrak{u}_{1}}}{\beta^{\mathfrak{s}}-1} \frac{\beta^{\mathfrak{d}_{1}}-1}{\beta^{\mathfrak{s}}-1}\right. \\
&\left.+\frac{\beta^{\mathfrak{u}_{1}}-1}{\beta^{\mathfrak{s}}-1} \frac{\beta^{\mathfrak{s}}-\beta^{\mathfrak{d}_{1}}}{\beta^{\mathfrak{s}}-1}+B_{\beta}^{*} \frac{\beta^{\mathfrak{u}_{1}}-1}{\beta^{\mathfrak{s}}-1} \frac{\beta^{\mathfrak{d}_{1}}-1}{\beta^{\mathfrak{s}}-1}\right),
\end{aligned}
$$

where

$$
\begin{aligned}
& A_{\beta}=\frac{G_{1}\left(o_{1}, o_{1}\right) G_{2}\left(o_{2}, o_{2}\right)}{\widetilde{G}(0,0)}=\frac{q r\left(\beta^{2}-1\right)}{(q \beta-1)(q r-1)}, \\
& B_{\beta}=\frac{(\beta-1)(q \beta+1)}{q \beta^{2}-1}, \quad \text { and } B_{\beta}^{*}=\frac{(\beta-1)(r \beta+1)}{r \beta^{2}-1} .
\end{aligned}
$$

(b) If $\alpha=1 / 2$ then

$$
G(x, y) \sim \frac{A_{1}}{\mathfrak{s}^{4} q^{\mathfrak{d}_{1}} r^{\mathfrak{d}_{2}}}\left(\frac{q+1}{q-1} \mathfrak{u}_{2} \mathfrak{d}_{2}+\mathfrak{s u}_{2} \mathfrak{d}_{1}+\mathfrak{s} \mathfrak{u}_{1} \mathfrak{d}_{2}+\frac{r+1}{r-1} \mathfrak{u}_{1} \mathfrak{d}_{1}\right),
$$

where

$$
A_{1}=\frac{G_{1}\left(o_{1}, o_{1}\right) G_{2}\left(o_{2}, o_{2}\right)}{2}=\frac{2 q r}{(q-1)(r-1)} .
$$

According to the way how $y$ tends to infinity geometrically (when we think of $x$ being fixed), one or more of the four terms will dominate the others, as we shall see below.

As mentioned at the beginning, the case $\alpha>1 / 2$ is obtained by exchanging $r \leftrightarrow q$ and $\alpha \leftrightarrow 1-\alpha$. Equivalently, we may use Lemma 4.12 and apply statement (a) of Theorem 4.2 to $G^{*}(x, y)$, with $\beta^{*}=1 / \beta$ in the place of $\beta$.

We now start our (laborious) way towards the proof of Theorem 4.2. The following is obvious, but crucial.

4.3. Lemma. The Green kernel $G(x, y)$ depends only on $\mathfrak{u}_{1}, \mathfrak{d}_{1}, \mathfrak{u}_{2}, \mathfrak{d}_{2}$.

Let $Z_{n}$ be the random position of the $P_{\alpha}$-walk. This is a DL-valued random variable defined on a suitable probability space (trajectory space). We write $\operatorname{Pr}_{x}=\operatorname{Pr}\left[\cdot \mid Z_{0}=x\right]$ and $\mathrm{E}_{x}$ for the associated expectation. Also, $\mathbf{1}_{[\cdots]}$ 
will denote the indicator function of an event $[\cdots]$ in the trajectory space. The projection $Z_{n}^{i}=\pi_{i}\left(Z_{n}\right)$ represents the random position at time $n$ of the $P_{i}$-walk on $\mathbb{T}^{i}, i=1,2$, and the random variable $\widetilde{Z}_{n}=\tilde{\pi}\left(Z_{n}\right)$ realizes the $n$-th position of the $\widetilde{P}_{\alpha}$-walk on $\mathbb{Z}$.

We shall use several stopping times. If $x=x_{1} x_{2} \in \mathrm{DL}, x_{i} \in \mathbb{T}^{i}(i=1,2)$, resp. $k \in Z$, then we set

$$
\begin{aligned}
& \mathbf{t}(x)=\inf \left\{n \geqslant 0: Z_{n}=x\right\}, \quad \mathbf{t}_{i}\left(x_{i}\right)=\inf \left\{n \geqslant 0: Z_{n}^{i}=x_{i}\right\} \quad(i=1,2), \quad \text { and } \\
& \tilde{\mathbf{t}}(k)=\inf \left\{n \geqslant 0: \widetilde{Z}_{n}=k\right\} .
\end{aligned}
$$

Note that these random variables are all defined on the same trajectory space associated with $P_{\alpha}$.

4.4. Lemma. Referring to the situation of Fig. 3, we have

$$
\mathbf{t}_{1}\left(c_{1}\right)=\tilde{\mathbf{t}}\left(\mathfrak{h}\left(x_{1}\right)-\mathfrak{u}_{1}\right) \quad \text { and } \quad \mathbf{t}_{2}\left(c_{2}\right)=\tilde{\mathbf{t}}\left(\mathfrak{h}\left(x_{2}\right)+\mathfrak{u}_{2}\right) \quad \operatorname{Pr}_{x} \text {-almost surely. }
$$

Furthermore, in order to reach y starting in $x$, both $\mathbb{Z}_{n}^{i}$ have to pass through $c_{i}, i=1,2$, i.e.,

$$
\max \left\{\mathbf{t}_{1}\left(c_{1}\right), \mathbf{t}_{2}\left(c_{2}\right)\right\} \leqslant \mathbf{t}(y) \quad \operatorname{Pr}_{x} \text {-almost surely. }
$$

Proof. The $P_{\alpha}$-walk on $\mathrm{DL}$ as well as the projected random walks are of nearest neighbour type. Thus, starting in $x$, the first point in the set $\left\{v=v_{1} v_{2} \in \mathrm{DL}: \mathfrak{h}\left(v_{1}\right)=\mathfrak{h}\left(c_{1}\right)\right\}$ visited by $Z_{n}$ must be of the form $c_{1} v_{2}$. This translates into $\mathbf{t}_{1}\left(c_{1}\right)=\tilde{\mathbf{t}}\left(\mathfrak{h}\left(x_{1}\right)-\mathfrak{u}_{1}\right)$, and exchanging the roles of the two trees, also into $\mathbf{t}_{2}\left(c_{2}\right)=\tilde{\mathbf{t}}\left(\mathfrak{h}\left(x_{2}\right)+\mathfrak{u}_{2}\right)$. The same type of argument shows the last statement.

The last lemma leads us to the identities

$$
\operatorname{Pr}_{x}\left[\mathbf{t}_{1}\left(c_{1}\right)<\mathbf{t}_{2}\left(c_{2}\right)\right]=\varphi_{1}\left(\mathfrak{u}_{1}, \mathfrak{u}_{2}\right) \quad \text { and } \quad \operatorname{Pr}_{x}\left[\mathbf{t}_{2}\left(c_{2}\right)<\mathbf{t}_{1}\left(c_{1}\right)\right]=\varphi_{2}\left(\mathfrak{u}_{1}, \mathfrak{u}_{2}\right),
$$

where for $k, l \geqslant 0$, the probability that the $\widetilde{P}_{\alpha}$-walk on $\mathbb{Z}$ starting in 0 reaches $-k$ before $l$ is $\varphi_{1}(k, l)$, and the probability that it reaches $l$ before $-k$ is $\varphi_{2}(k, l)=1-\varphi_{1}(k, l)$. It is a well-known exercise to compute these functions, and they are given by

$$
\begin{aligned}
& \varphi_{1}(k, l)=\frac{\beta^{k+l}-\beta^{k}}{\beta^{k+l}-1} \quad \text { and } \quad \varphi_{2}(k, l)=\frac{\beta^{k}-1}{\beta^{k+l}-1}, \quad \text { with } \beta=\frac{1-\alpha}{\alpha}, \text { if } \alpha \neq \frac{1}{2} ; \\
& \varphi_{1}(k, l)=\frac{l}{k+l} \quad \text { and } \quad \varphi_{2}(k, l)=\frac{k}{k+l}, \quad \text { if } \alpha=\frac{1}{2} .
\end{aligned}
$$

See e.g. Kemeny and Snell [19], §7.1, in particular (5) and (6) on p. 153. Next, let us introduce the function

$$
\psi(k)=\left(\frac{\alpha r}{(1-\alpha) q}\right)^{k}, \quad k \in \mathbb{Z} .
$$

If we set $\mathrm{m}(x)=\psi\left(\mathfrak{h}\left(x_{1}\right)\right)$, where $x=x_{1} x_{2} \in \mathrm{DL}$, then we have $\mathrm{m}(x) p_{\alpha}(x, y)=\mathrm{m}(y) p_{\alpha}(y, x)$ for all $x, y \in \mathrm{DL}$. That is, $P_{\alpha}$ is m-reversible, and we also get

$$
G(x, y)=\psi\left(\mathfrak{h}\left(y_{1}\right)-\mathfrak{h}\left(x_{1}\right)\right) G(y, x) \quad \text { for all } x=x_{1} x_{2}, y=y_{1} y_{2} \in \mathrm{DL} .
$$

4.9. Proposition. Referring to the situation of Fig. 3, we have the following decomposition.

$$
\begin{aligned}
G(x, y)= & \varphi_{1}\left(\mathfrak{u}_{1}, \mathfrak{u}_{2}\right) \varphi_{1}\left(\mathfrak{d}_{1}, \mathfrak{d}_{2}\right) \psi\left(\mathfrak{d}_{1}\right) G\left(c_{1} b_{2}, c_{1} a_{2}\right) \\
& +\varphi_{1}\left(\mathfrak{u}_{1}, \mathfrak{u}_{2}\right) \varphi_{2}\left(\mathfrak{d}_{1}, \mathfrak{d}_{2}\right) \psi\left(\mathfrak{d}_{1}\right) G\left(b_{1} c_{2}, c_{1} a_{2}\right) \\
& +\varphi_{2}\left(\mathfrak{u}_{1}, \mathfrak{u}_{2}\right) \varphi_{1}\left(\mathfrak{d}_{1}, \mathfrak{d}_{2}\right) \psi\left(-\mathfrak{d}_{2}\right) G\left(c_{1} b_{2}, a_{1} c_{2}\right) \\
& +\varphi_{2}\left(\mathfrak{u}_{1}, \mathfrak{u}_{2}\right) \varphi_{2}\left(\mathfrak{d}_{1}, \mathfrak{d}_{2}\right) \psi\left(-\mathfrak{d}_{2}\right) G\left(b_{1} c_{2}, a_{1} c_{2}\right) .
\end{aligned}
$$




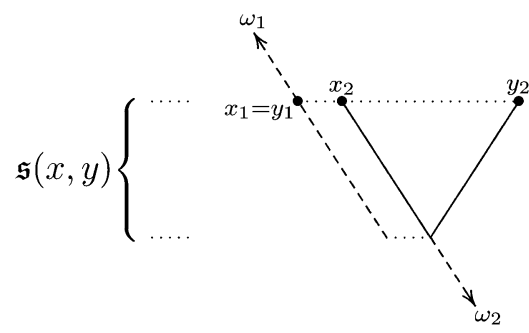

(I)

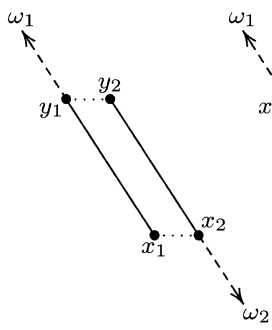

(II)

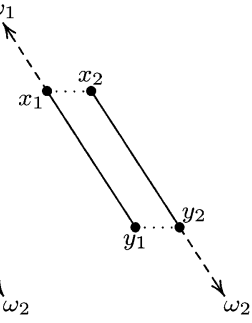

(III)

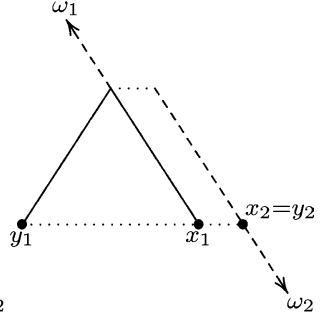

(IV)

Fig. 4.

Proof. By (4.4) and (4.5), we have

$$
G(x, y)=\mathrm{E}_{x}\left(\mathbf{1}_{\left[\mathbf{t}_{1}\left(c_{1}\right)<\mathbf{t}_{2}\left(c_{2}\right)\right]} G\left(Z_{\mathbf{t}_{1}\left(c_{1}\right)}, y\right)\right)+\mathrm{E}_{x}\left(\mathbf{1}_{\left[\mathbf{t}_{2}\left(c_{2}\right)<\mathbf{t}_{1}\left(c_{1}\right)\right]} G\left(Z_{\mathbf{t}_{2}\left(c_{2}\right)}, y\right)\right) .
$$

If $\mathbf{t}_{1}\left(c_{1}\right)<\mathbf{t}_{2}\left(c_{2}\right)$ and $Z_{\mathbf{t}_{1}\left(c_{1}\right)}=c_{1} w_{2}$, then we must have $c_{2} \preccurlyeq w_{2}$ and furthermore $w_{2} \curlywedge b_{2}=c_{2}$. Thus, $\mathfrak{u}\left(w_{2}, y_{2}\right)=\mathfrak{u}\left(a_{2}, y_{2}\right)$ and $\mathfrak{d}\left(w_{2}, y_{2}\right)=\mathfrak{d}\left(a_{2}, y_{2}\right)$. Lemma 4.3 implies $G\left(Z_{\mathbf{t}_{1}\left(c_{1}\right)}, y\right)=G\left(c_{1} a_{2}, y\right)$. In the same way, $G\left(Z_{\mathbf{t}_{2}\left(c_{2}\right)}, y\right)=G\left(a_{1} c_{2}, y\right)$. Thus,

$$
\begin{aligned}
G(x, y) & =\operatorname{Pr}_{x}\left[\mathbf{t}_{1}\left(c_{1}\right)<\mathbf{t}_{2}\left(c_{2}\right)\right] G\left(c_{1} a_{2}, y\right)+\operatorname{Pr}_{x}\left[\mathbf{t}_{2}\left(c_{2}\right)<\mathbf{t}_{1}\left(c_{1}\right)\right] G\left(a_{1} c_{2}, y\right) \\
& =\varphi_{1}\left(\mathfrak{u}_{1}, \mathfrak{u}_{2}\right) G\left(c_{1} a_{2}, y\right)+\varphi_{2}\left(\mathfrak{u}_{1}, \mathfrak{u}_{2}\right) G\left(a_{1} c_{2}, y\right) .
\end{aligned}
$$

Using (4.8), we get $G\left(c_{1} a_{2}, y\right)=\psi\left(\mathfrak{d}_{1}\right) G\left(y, c_{1} a_{2}\right)$. Applying once more (4.4) and (4.5),

$$
G\left(y, c_{1} a_{2}\right)=\mathrm{E}_{y}\left(\mathbf{1}_{\left[\mathbf{t}_{1}\left(c_{1}\right)<\mathbf{t}_{2}\left(c_{2}\right)\right]} G\left(Z_{\mathbf{t}_{1}\left(c_{1}\right)}, y\right)\right)+\mathrm{E}_{y}\left(\mathbf{1}_{\left[\mathbf{t}_{2}\left(c_{2}\right)<\mathbf{t}_{1}\left(c_{1}\right)\right]} G\left(Z_{\mathbf{t}_{2}\left(c_{2}\right)}, y\right)\right) .
$$

We can repeat the above argument with $y$ in the place of $x$ and $c_{1} a_{2}$ in the place of $y$, and we have to replace $a_{1}, a_{2}$ with $b_{1}, b_{2}$. Therefore

$$
G\left(y, c_{1} a_{2}\right)=\varphi_{1}\left(\mathfrak{d}_{1}, \mathfrak{d}_{2}\right) G\left(c_{1} b_{2}, c_{1} a_{2}\right)+\varphi_{2}\left(\mathfrak{d}_{1}, \mathfrak{d}_{2}\right) G\left(b_{1} c_{2}, c_{1} a_{2}\right) .
$$

Analogously, $G\left(a_{1} c_{2}, y\right)=\psi\left(-\mathfrak{d}_{2}\right) G\left(y, a_{1} c_{2}\right)$ and

$$
G\left(y, a_{1} c_{2}\right)=\varphi_{1}\left(\mathfrak{d}_{1}, \mathfrak{d}_{2}\right) G\left(c_{1} b_{2}, a_{1} c_{2}\right)+\varphi_{2}\left(\mathfrak{d}_{1}, \mathfrak{d}_{2}\right) G\left(b_{1} c_{2}, a_{1} c_{2}\right) .
$$

Combining these formulas, we obtain the proposed decomposition.

Thus, in order to understand the asymptotics of $G(x, y)$ in the general case of Fig. 3, we can reduce our computations to the following four basic cases of relative positions of $x$ and $y$.

In all four cases, $\mathfrak{s}=\mathfrak{s}(x, y) \rightarrow \infty$. In case (I), $\mathfrak{u}_{1}=\mathfrak{d}_{1}=0$ and $\mathfrak{u}_{2}=\mathfrak{d}_{2}=\mathfrak{s}$. In case (II), $\mathfrak{u}_{1}=\mathfrak{d}_{2}=\mathfrak{s}$ and $\mathfrak{d}_{1}=\mathfrak{u}_{2}=0$. In case (III), $\mathfrak{d}_{1}=\mathfrak{u}_{2}=\mathfrak{s}$ and $\mathfrak{u}_{1}=\mathfrak{d}_{2}=0$. In case (IV), $\mathfrak{u}_{1}=\mathfrak{d}_{1}=\mathfrak{s}$ and $\mathfrak{u}_{2}=\mathfrak{d}_{2}=0$.

We start with an extended version of case II, see Fig. 5.

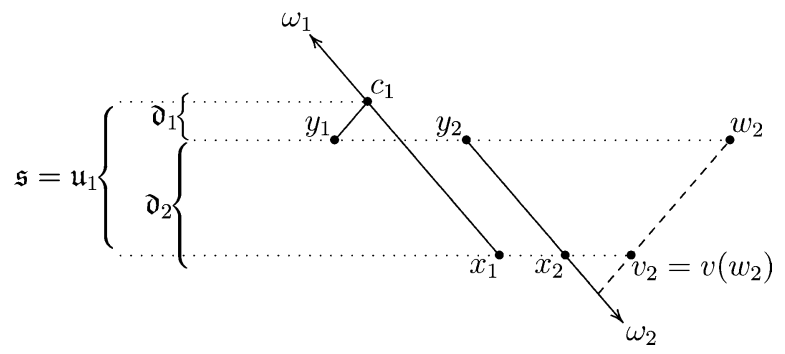

Fig. 5 . 
4.10. Proposition. If, as in Fig. 5, $x=x_{1} x_{2}$ and $y=y_{1} y_{2}$ with $\mathfrak{s}=\mathfrak{s}(x, y)$ are such that $\mathfrak{u}_{1}-\mathfrak{d}_{1}=\mathfrak{d}_{2} \rightarrow \infty, \mathfrak{u}_{2}=0$ and $\mathfrak{d}_{1}$ is arbitrary, then

$$
G_{1}\left(x_{1}, y_{1}\right)=C(\mathfrak{s}) r^{\mathfrak{d}_{2}} G(x, y)+R\left(\mathfrak{d}_{1}, \mathfrak{d}_{2}\right),
$$

where

$$
C(\mathfrak{s}) \rightarrow \frac{\widetilde{G}(0,0)}{G_{2}\left(o_{2}, o_{2}\right)} \quad \text { if } \alpha \neq \frac{1}{2}, \quad C(\mathfrak{s}) \sim \frac{2 \mathfrak{s}}{G_{2}\left(o_{2}, o_{2}\right)} \quad \text { if } \alpha=\frac{1}{2},
$$

and $0<R\left(\mathfrak{d}_{1}, \mathfrak{d}_{2}\right)<G_{1}\left(x_{1}, y_{1}\right)$ with

$$
\lim _{\mathfrak{d}_{2} \rightarrow \infty} R\left(\mathfrak{d}_{1}, \mathfrak{d}_{2}\right)=0 \text {. }
$$

Proof. Applying (3.1) to the projection $\pi_{1}$ gives $G_{1}\left(x_{1}, y_{1}\right)=\sum_{w_{2} \in H\left(y_{2}\right)} G\left(x, y_{1} w_{2}\right)$.

Let $w_{2} \in H\left(y_{2}\right)$, where $H\left(y_{2}\right)$ is the horocycle of $y_{2}$ in $\mathbb{T}_{r}$. We write $v_{2}=v\left(w_{2}\right)$ for the unique element in $H\left(x_{2}\right)$ that satisfies $v_{2} \preccurlyeq w_{2}$. By Lemma 4.4, the random walk has to pass through some point of the form in $\left\{u_{1} v_{2}: u_{1} \in H\left(x_{1}\right)\right\}$ on the way from $x$ to $y_{1} w_{2}$, that is,

$$
\begin{aligned}
G\left(x, y_{1} w_{2}\right) & =\mathrm{E}_{x}\left(\mathbf{1}_{\left[\mathbf{t}_{2}\left(v_{2}\right)<\infty\right]} G\left(Z_{\mathbf{t}_{2}\left(v_{2}\right)}, y_{1} w_{2}\right)\right) \\
& =\mathrm{E}_{x}\left(\mathbf{1}_{\left[\mathbf{t}_{2}\left(v_{2}\right)<\mathbf{t}_{1}\left(c_{1}\right)\right]} G\left(Z_{\mathbf{t}_{2}\left(v_{2}\right)}, y_{1} w_{2}\right)\right)+\mathrm{E}_{x}\left(\mathbf{1}_{\left[\mathbf{t}_{1}\left(c_{1}\right)<\mathbf{t}_{2}\left(v_{2}\right)<\infty\right]} G\left(Z_{\mathbf{t}_{2}\left(v_{2}\right)}, y_{1} w_{2}\right)\right) .
\end{aligned}
$$

Now, if starting at $x$, we have $\mathbf{t}_{2}\left(v_{2}\right)<\mathbf{t}_{1}\left(c_{1}\right)$, then $Z_{\mathbf{t}_{2}\left(v_{2}\right)}=u_{1} v_{2}$ for some random $u_{1} \in H\left(x_{1}\right)$ that must satisfy $\mathfrak{u}\left(u_{1}, y_{1}\right)=\mathfrak{u}_{1}$ and $\mathfrak{d}\left(u_{1}, y_{1}\right)=\mathfrak{d}_{1}$, since $c_{1}$ cannot lie on $\overline{x_{1} u_{1}}$. But we also have $\mathfrak{u}\left(v_{2}, w_{2}\right)=\mathfrak{u}_{2}=0$ and $\mathfrak{d}\left(v_{2}, w_{2}\right)=\mathfrak{d}_{2}$. That is, the points $u_{1} v_{2}$ and $y_{1} w_{2}$ have the same relative position as the points $x$ and $y$, and therefore $G\left(u_{1} v_{2}, y_{1} w_{2}\right)=G(x, y)$ by Lemma 4.3. We get

$$
\mathrm{E}_{x}\left(\mathbf{1}_{\left[\mathbf{t}_{2}\left(v_{2}\right)<\mathbf{t}_{1}\left(c_{1}\right)\right]} G\left(Z_{\mathbf{t}_{2}\left(v_{2}\right)}, y_{1} w_{2}\right)\right)=\operatorname{Pr}_{x}\left[\mathbf{t}_{2}\left(v_{2}\right)<\mathbf{t}_{1}\left(c_{1}\right)\right] G(x, y) .
$$

Now, given $v_{2} \in H\left(x_{2}\right)$, there are precisely $r^{\mathfrak{d}_{2}}$ elements $w_{2} \in H\left(y_{2}\right)$ with $v\left(w_{2}\right)=v_{2}$. Combining all these observations,

$$
\begin{aligned}
& G_{1}\left(x_{1}, y_{1}\right)=\left(\sum_{v_{2} \in H\left(x_{2}\right)} \operatorname{Pr}_{x}\left[\mathbf{t}_{2}\left(v_{2}\right)<\mathbf{t}_{1}\left(c_{1}\right)\right]\right) r^{\mathfrak{d}_{2}} G(x, y)+R\left(\mathfrak{d}_{1}, \mathfrak{d}_{2}\right), \quad \text { where } \\
& R\left(\mathfrak{d}_{1}, \mathfrak{d}_{2}\right)=\sum_{w_{2} \in H\left(y_{2}\right)} \mathrm{E}_{x}\left(\mathbf { 1 } _ { [ \mathbf { t } _ { 1 } ( c _ { 1 } ) < \mathbf { t } _ { 2 } ( v ( w _ { 2 } ) ) < \infty ] } G \left(Z_{\left.\left.\mathbf{t}_{2}\left(v\left(w_{2}\right)\right), y_{1} w_{2}\right)\right) .}\right.\right.
\end{aligned}
$$

Let us first consider the error term $R\left(\mathfrak{d}_{1}, \mathfrak{d}_{2}\right)$. Note that $G(\cdot, \cdot) \leqslant G(o, o)<\infty$, since our random walk is transient. (Already the projections onto $\mathbb{T}_{q}$ and $\mathbb{T}_{r}$ are transient!) Since $\mathfrak{d}_{2} \rightarrow \infty$, also $\mathbf{t}_{1}\left(c_{1}\right) \rightarrow \infty$ almost surely. It follows that

$$
\begin{aligned}
r_{\mathfrak{d}_{1}, \mathfrak{d}_{2}}\left(w_{2}\right): & :=\mathrm{E}_{x}\left(\mathbf{1}_{\left[\mathbf{t}_{1}\left(c_{1}\right)<\mathbf{t}_{2}\left(v\left(w_{2}\right)\right)<\infty\right]} G\left(Z_{\mathbf{t}_{2}\left(v\left(w_{2}\right)\right)}, y_{1} w_{2}\right)\right) \\
& \leqslant \operatorname{Pr}_{x}\left[\mathbf{t}_{1}\left(c_{1}\right)<\mathbf{t}_{2}\left(v\left(w_{2}\right)\right)<\infty\right] G(o, o) \rightarrow 0 \text { when } \mathfrak{d}_{2} \rightarrow \infty .
\end{aligned}
$$

On the other hand,

$$
r_{\mathfrak{d}_{1}, \mathfrak{d}_{2}}\left(w_{2}\right) \leqslant G\left(x, y_{1} w_{2}\right) \text { and } \sum_{w_{2} \in H\left(y_{2}\right)} G\left(x, y_{1} w_{2}\right)=G_{1}\left(x_{1}, y_{1}\right) \leqslant G_{1}\left(o_{1}, o_{1}\right) .
$$

Thus, dominated convergence (in the summation) implies that $R\left(\mathfrak{d}_{1}, \mathfrak{d}_{2}\right) \rightarrow 0$ as $\mathfrak{d}_{2} \rightarrow \infty$.

It remains to show that $C(\mathfrak{s})=\sum_{v_{2} \in H\left(x_{2}\right)} \operatorname{Pr}_{x}\left[\mathbf{t}_{2}\left(v_{2}\right)<\mathbf{t}_{1}\left(c_{1}\right)\right]$ has the proposed asymptotic behaviour, when $\mathfrak{d}_{2}$ (and $\mathfrak{s}) \rightarrow \infty$.

We may suppose without loss of generality that $\mathfrak{h}\left(x_{1}\right)=\mathfrak{h}\left(x_{2}\right)=0$, so that $\mathfrak{h}\left(c_{1}\right)=-\mathfrak{s}$. Then Lemma 4.4 implies $\operatorname{Pr}_{x}\left[\mathbf{t}_{2}\left(v_{2}\right)<\mathbf{t}_{1}\left(c_{1}\right)\right]=\operatorname{Pr}_{x}\left[\mathbf{t}_{2}\left(v_{2}\right)<\tilde{\mathbf{t}}(-\mathfrak{s})\right]$. Now let the superscript ${ }^{(-\mathfrak{s})}$ refer to the random walk $\widetilde{P}$ on $\mathbb{Z}$ stopped 


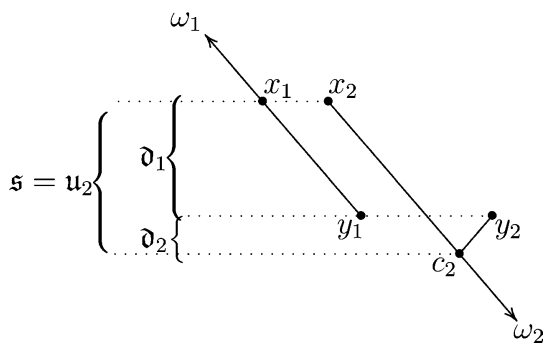

Fig. 6 .

at $-\mathfrak{s}$, i.e., we consider the restriction of $\widetilde{P}$ to $\{k \in \mathbb{Z}: k>-\mathfrak{s}\}$. We use the same superscript for the random walk $P_{2}$ on $\mathbb{T}_{r}$ stopped at the horocycle $H_{\mathfrak{s}}$ in $\mathbb{T}_{r}$, i.e., we consider the restriction of $P_{2}$ to $\left\{z_{2} \in \mathbb{T}_{r}: \mathfrak{h}\left(z_{2}\right)<\mathfrak{s}\right\}$. Using the notation of (3.5), we have

$$
\operatorname{Pr}_{x}\left[\mathbf{t}_{2}\left(v_{2}\right)<\tilde{\mathbf{t}}(-\mathfrak{s})\right]=F_{2}^{(-\mathfrak{s})}\left(x_{2}, v_{2}\right)=\frac{G_{2}^{(-\mathfrak{s})}\left(x_{2}, v_{2}\right)}{G_{2}^{(-\mathfrak{s})}\left(v_{2}, v_{2}\right)}=\frac{G_{2}^{(-\mathfrak{s})}\left(x_{2}, v_{2}\right)}{G_{2}^{(-\mathfrak{s})}\left(o_{2}, o_{2}\right)}
$$

Since $\widetilde{P}^{(-\mathfrak{s})}$ is the projection of $P_{2}(-\mathfrak{s})$ under the mapping $z_{2} \mapsto-\mathfrak{h}\left(z_{2}\right)$, we find

$$
C(\mathfrak{s})=\sum_{v_{2} \in H\left(x_{2}\right)} \frac{G_{2}^{(-\mathfrak{s})}\left(x_{2}, v_{2}\right)}{G_{2}^{(-\mathfrak{s})}\left(o_{2}, o_{2}\right)}=\frac{\widetilde{G}^{(-\mathfrak{s})}(0,0)}{G_{2}^{(-\mathfrak{s})}\left(o_{2}, o_{2}\right)} .
$$

If $\mathfrak{s} \rightarrow \infty$ then $G_{2}^{(-\mathfrak{s})}\left(o_{2}, o_{2}\right) \rightarrow G_{2}\left(o_{2}, o_{2}\right)<\infty$, for each value of $\alpha$. If $\alpha<1 / 2$ then $\widetilde{G}^{(-\mathfrak{s})}(0,0) \rightarrow \widetilde{G}(0,0)<$ $\infty$. If $\alpha=1 / 2$ then routine calculations regarding SRW on $\mathbb{Z}$ yield $\widetilde{G}^{(-\mathfrak{s})}(0,0)=2 \mathfrak{s}$. Thus, $C(\mathfrak{s})$ has the proposed asymptotic behaviour.

The last proposition is valid for arbitrary $\alpha$. However, it becomes meaningful only when $\alpha \leqslant 1 / 2$. Indeed, when $\alpha>1 / 2$, then the left-hand side in the decomposition (4.11) tends to 0 by (3.5). In this case, (4.11) contains no information about the asymptotic behaviour of $G(x, y)$. On the other hand, when $\alpha \leqslant 1 / 2$ and $\mathfrak{d}_{1}=0$ (situation (II) of Fig. 4) then $G_{1}\left(x_{1}, y_{1}\right)=G_{1}\left(o_{1}, o_{1}\right)$ is constant, see (3.5). When we consider the "dual" situation of Fig. 5, as illustrated in Fig. 6, this discussion shows that it is not useful to rewrite Proposition 4.10 by just exchanging both the roles of the two trees and $\alpha$ with $1-\alpha$.

We shall use the superscript ${ }^{*}$ for the respective random walks on DL, $\mathbb{T}_{q}, \mathbb{T}_{r}$, and $\mathbb{Z}$ that are obtained by exchanging $\alpha \leftrightarrow 1-\alpha$, without exchanging roles of the two trees. Thus, $P_{\alpha}^{*}=P_{1-\alpha}, P_{\alpha, q}^{*}=P_{1-\alpha, q}, P_{1-\alpha, r}^{*}=P_{\alpha, r}$, and $\widetilde{P}^{*}$ on $\mathbb{Z}$ moves from $k$ to $k+1$ with probability $1-\alpha$ and to $k-1$ with probability $\alpha$.

\subsection{Lemma.}

$$
G^{*}(x, y)=\beta^{\mathfrak{h}\left(y_{1}\right)-\mathfrak{h}\left(x_{1}\right)} G(x, y) \quad \forall x, y \in \mathrm{DL}\left(\beta=\frac{1-\alpha}{\alpha}\right) .
$$

Proof. The function $g(x)=\beta^{\mathfrak{h}\left(x_{1}\right)}$ satisfies $P g=g$, and $p^{*}(x, y)=p(x, y) g(y) / g(x)$.

4.13. Corollary. If, as in Fig. 6, $x=x_{1} x_{2}$ and $y=y_{1} y_{2}$ with $\mathfrak{s}=\mathfrak{s}(x, y)$ are such that $\mathfrak{d}_{1}=\mathfrak{u}_{2}-\mathfrak{d}_{2} \rightarrow \infty$, $\mathfrak{u}_{1}=0$ and $\mathfrak{d}_{2}$ is arbitrary, then

$$
G_{2}^{*}\left(x_{2}, y_{2}\right)=C^{*}(\mathfrak{s})(q \beta)^{\mathfrak{d}_{1}} G(x, y)+R^{*}\left(\mathfrak{d}_{1}, \mathfrak{d}_{2}\right),
$$


where

$$
C^{*}(\mathfrak{s}) \rightarrow \frac{\widetilde{G}(0,0)}{G_{1}\left(o_{1}, o_{1}\right)} \quad \text { if } \alpha \neq \frac{1}{2}, \quad C^{*}(\mathfrak{s}) \sim \frac{2 \mathfrak{s}}{G_{1}\left(o_{1}, o_{1}\right)} \quad \text { if } \alpha=\frac{1}{2},
$$

and $0<R^{*}\left(\mathfrak{d}_{1}, \mathfrak{d}_{2}\right)<G_{2}^{*}\left(x_{2}, y_{2}\right)$ with

$$
\lim _{\mathfrak{d}_{1} \rightarrow \infty} R^{*}\left(\mathfrak{d}_{1}, \mathfrak{d}_{2}\right)=0
$$

This is immediate by applying Proposition 4.10 to $P^{*}$ with $r \leftrightarrow q$. Also observe that $\widetilde{G}^{*}(0,0)=\widetilde{G}(0,0)$ and $G_{i}^{*}\left(o_{i}, o_{i}\right)=G_{i}\left(o_{i}, o_{i}\right)$ for $i=1,2$. In the specific case $\mathfrak{d}_{1}=0$ (resp. $\mathfrak{d}_{2}=0$ ), Proposition 4.10 (resp. Corollary 4.13) yields the asymptotic behaviour of $G(x, y)$ in situation (II) (resp. (III)) of Fig. 4.

4.15. Corollary. (a) Referring to situation (II) of Fig. 4 , if $\mathfrak{s}=\mathfrak{u}_{1}=\mathfrak{d}_{2} \rightarrow \infty$ and $\mathfrak{d}_{1}=\mathfrak{u}_{2}=0$ then

$$
G(x, y) \sim \begin{cases}\frac{G_{1}\left(o_{1}, o_{1}\right) G_{2}\left(o_{2}, o_{2}\right)}{\widetilde{G}(0,0) r^{\mathfrak{s}}} & \text { if } \alpha>1 / 2, \quad \text { and } \\ \frac{G_{1}\left(o_{1}, o_{1}\right) G_{2}\left(o_{2}, o_{2}\right)}{2 \mathfrak{s} r^{\mathfrak{s}}} & \text { if } \alpha=1 / 2 .\end{cases}
$$

(b) Referring to situation (III) of Fig. 4, if $\mathfrak{s}=\mathfrak{d}_{1}=\mathfrak{u}_{2} \rightarrow \infty$ and $\mathfrak{u}_{1}=\mathfrak{d}_{2}=0$ then

$$
G(x, y) \sim \begin{cases}\frac{G_{1}\left(o_{1}, o_{1}\right) G_{2}\left(o_{2}, o_{2}\right)}{\widetilde{G}(0,0)(q \beta)^{\mathfrak{s}}} & \text { if } \alpha>1 / 2, \quad \text { and } \\ \frac{G_{1}\left(o_{1}, o_{1}\right) G_{2}\left(o_{2}, o_{2}\right)}{2 \mathfrak{s} q^{\mathfrak{s}}} & \text { if } \alpha=1 / 2 .\end{cases}
$$

Proposition 4.10, resp. Corollary 4.13, also leads to an asymptotic estimate of $G(x, y)$ when $\mathfrak{d}_{1}$, resp. $\mathfrak{d}_{2}$, remains bounded. Otherwise, the left-hand side of the decomposition (4.11), resp. (4.14), tends to 0. Nevertheless, those decompositions will now be useful "on the average" for situations (I) and (IV) of Fig. 4.

4.16. Proposition. Referring to situation (IV) of Fig. 4, if $x=x_{1} x_{2}$ and $y=y_{1} y_{2}$ with $\mathfrak{s}=\mathfrak{s}(x, y)$ are such that $\mathfrak{u}_{1}=\mathfrak{d}_{1}=\mathfrak{s} \rightarrow \infty$ and $\mathfrak{u}_{2}=\mathfrak{d}_{2}=0\left(y_{2}=x_{2}\right)$ then

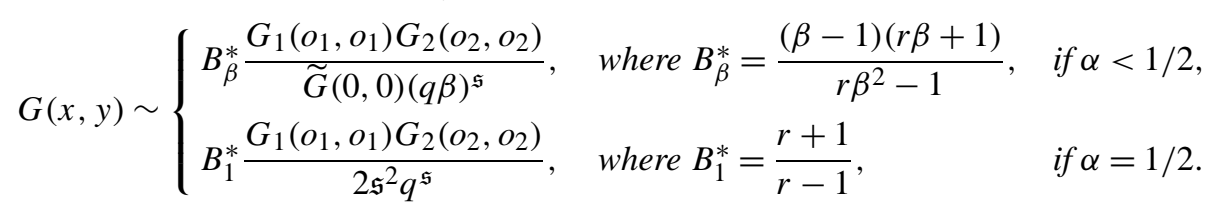

Proof. Again, we may assume that $\mathfrak{h}\left(x_{1}\right)=\mathfrak{h}\left(x_{2}\right)=0$. Since $1-\alpha \geqslant 1 / 2$,

$$
\mathbf{t}:=\mathbf{t}_{1}\left(c_{1}\right)=\tilde{\mathbf{t}}(-\mathfrak{s})<\infty \quad \operatorname{Pr}_{x} \text {-almost surely. }
$$

This and Lemma 4.4 yield

$$
G(x, y)=\mathrm{E}_{x}\left(\mathbf{1}_{[\mathbf{t}<\infty]} G\left(Z_{\mathbf{t}}, y\right)\right)=\mathrm{E}_{x}\left(G\left(Z_{\mathbf{t}}, y\right)\right) .
$$

We have $\pi_{1}\left(Z_{\mathbf{t}}\right)=c_{1}$. Set $D_{\mathbf{t}}=\mathfrak{d}\left(Z_{\mathbf{t}}^{2}, x_{2}\right)$, a non-negative, integer-valued random variable, see Fig. 7 .

The relative position of $c_{1} Z_{\mathfrak{t}}^{2}$ (in the place of $x$ ) and $y=y_{1} x_{2}$ is precisely the one of Fig. 6 , replacing $\mathfrak{s}=\mathfrak{s}(x, y)$ with $\mathfrak{s}+D_{\mathbf{t}}$ and $\mathfrak{d}_{2}$ with $D_{\mathbf{t}}$. We can apply Corollary 4.13. Note that by (3.5), applied to $P_{2}^{*}=P_{\alpha, r}$,

$$
G_{2}^{*}\left(Z_{\mathbf{t}}^{2}, x_{2}\right)=(r \beta)^{-D_{\mathbf{t}}} G_{2}^{*}\left(x_{2}, x_{2}\right)=(r \beta)^{-D_{\mathbf{t}}} G_{2}\left(o_{2}, o_{2}\right) .
$$

We get 


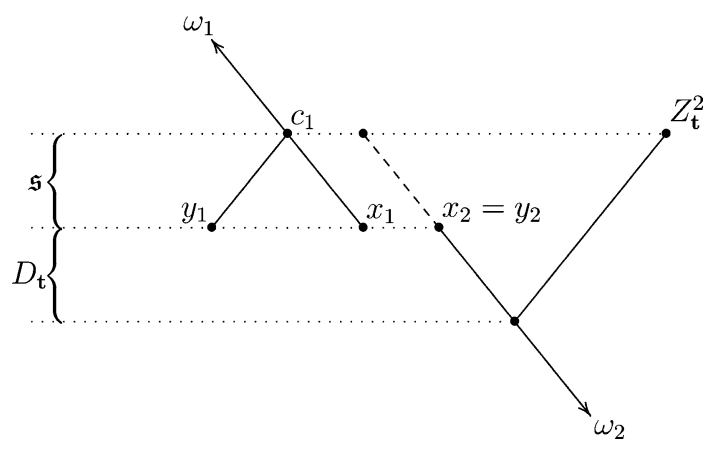

Fig. 7.

$$
\begin{aligned}
G\left(Z_{\mathbf{t}}, y\right) & =\frac{1}{C^{*}\left(\mathfrak{s}+D_{\mathfrak{t}}\right)(q \beta)^{\mathfrak{s}}}\left(G_{2}^{*}\left(Z_{\mathbf{t}}^{2}, x_{2}\right)-R^{*}\left(\mathfrak{s}, D_{\mathbf{t}}\right)\right) \\
& =\frac{1}{C^{*}\left(\mathfrak{s}+D_{\mathfrak{t}}\right)(q \beta)^{\mathfrak{s}}}\left(\frac{1}{(r \beta)^{D_{\mathfrak{t}}}} G_{2}\left(o_{2}, o_{2}\right)-R^{*}\left(\mathfrak{s}, D_{\mathbf{t}}\right)\right) .
\end{aligned}
$$

Case 1. $\alpha<1 / 2$. Then $C^{*}\left(\mathfrak{s}+D_{\mathfrak{t}}\right) \rightarrow \widetilde{G}(0,0) / G_{1}\left(o_{1}, o_{1}\right)$, a finite limit. Since $R^{*}\left(\mathfrak{s}, D_{\mathfrak{t}}\right)<G_{2}^{*}\left(Z_{\mathfrak{t}}^{2}, x_{2}\right) \leqslant$ $G_{2}^{*}\left(o_{2}, o_{2}\right)$ and $R^{*}\left(\mathfrak{s}, D_{\mathfrak{t}}\right) \rightarrow 0$ as $\mathfrak{s} \rightarrow \infty$, dominated convergence yields

$$
\mathrm{E}_{x}\left[R^{*}\left(\mathfrak{s}, D_{\mathfrak{t}}\right)\right] \rightarrow 0 .
$$

Also, $Z_{n}^{2}$ converges almost surely to a $\partial^{*} \mathbb{T}_{r}$-valued random variable $Z_{\infty}^{2}$; see Cartwright, Kaimanovich and Woess [5], where this is proved under much more general assumptions. Since $\mathbf{t} \rightarrow \infty$ when $\mathfrak{s} \rightarrow \infty$, we get $Z_{\mathbf{t}}^{2} \rightarrow Z_{\infty}^{2}$ and consequently

$$
D_{\mathbf{t}} \rightarrow D_{\infty}=d\left(o_{2}, o_{2} \curlywedge Z_{2}^{\infty}\right) .
$$

(Cf. Section 2 for notation.) Therefore, (4.17) and (4.18) yield

$$
\mathrm{E}_{x}\left(G\left(Z_{\mathbf{t}}, y\right)\right) \sim \frac{G_{1}\left(o_{1}, o_{1}\right) G_{2}\left(o_{2}, o_{2}\right)}{\widetilde{G}(0,0)(q \beta)^{\mathfrak{s}}} B_{\beta}^{*} \quad \text { as } \mathfrak{s} \rightarrow \infty
$$

where $B_{\beta}^{*}=\mathrm{E}_{x}\left[(r \beta)^{-D_{\infty}}\right]$. This number can be computed explicitly: let $w_{2}^{(m)}$ denote the vertex on $\overline{x_{2} \omega_{2}}$ at distance $m$ from $x_{2}$. If $m \geqslant 1$, then $D_{\infty} \geqslant m$ precisely when $Z_{\infty}^{2} \in \widehat{\mathbb{T}}_{r}\left(x_{2}, w_{2}^{(m)}\right)$. Applying a frequently used formula for the limit distribution on the boundary of arbitrary transient nearest neighbour random walks on trees (see e.g. [4]), we get that

$$
\operatorname{Pr}_{x}\left[D_{\infty} \geqslant m\right]=\frac{F_{2}\left(x_{2}, w_{2}^{(m)}\right)\left(1-F_{2}\left(w_{2}^{(m)}, w_{2}^{(m-1)}\right)\right)}{1-F_{2}\left(w_{2}^{(m-1)}, w_{2}^{(m)}\right) F_{2}\left(w_{2}^{(m)}, w_{2}^{(m-1)}\right)}=\beta^{-m} \frac{\beta r-\beta}{\beta r-1} .
$$

We have used the $P_{2}$-version of (3.5) in the last computation. It is now straightforward that $B_{\beta}^{*}$ has the proposed value.

Case 2. $\alpha=1 / 2$. Here, we need to compute explicitly the distribution of $D_{\mathfrak{t}}$, which depends on $\mathfrak{s}$. Consider the random variable $M=M_{\mathfrak{s}}=\max \left\{\widetilde{Z}_{n}: n<\mathbf{t}\right\}$. If $\mathfrak{n}=\mathfrak{n}_{\mathfrak{s}}=\max \left\{n<\mathbf{t}: \widetilde{Z}_{n}=M_{\mathfrak{s}}\right\}$, then $Z_{\mathfrak{n}}^{2} \operatorname{must}$ be the point $w_{2}=w_{2}^{(M)}$ on $\overline{o_{2} \omega_{2}}$.

Conditioned on the value of $M_{\mathfrak{s}}$, the random element $Z_{\mathfrak{t}}^{2}$ is equidistributed on the set $\left\{v_{2} \in \mathbb{T}_{r}: \mathfrak{h}\left(v_{2}\right)=\mathfrak{s}\right.$, $\left.w_{2} \preccurlyeq v_{2}\right\}$, which has $r^{\mathfrak{s}+M}$ elements. Among the latter, the number of elements with $\mathfrak{d}\left(v_{2}, x_{2}\right)=d \in\{0, \ldots, M\}$ is $r^{\mathfrak{s}}$, if $d=0$, and $(r-1) r^{\mathfrak{s}+d-1}$, if $d \geqslant 1$. If $D_{\mathfrak{t}}=d$ then $M_{\mathfrak{s}} \geqslant d$. Thus, if $0 \leqslant d \leqslant m$ then

$$
\operatorname{Pr}_{x}\left[D_{\mathbf{t}}=d \mid M=m\right]=\epsilon_{d} r^{d-m}, \quad \text { where } \epsilon_{d}= \begin{cases}1 & \text { if } d=0, \\ (r-1) / r & \text { if } d \geqslant 1 .\end{cases}
$$


Also, $\operatorname{Pr}_{x}[M \geqslant m]$ is the probability that the random walk $\widetilde{Z}_{n}$ on $\mathbb{Z}$ reaches $m$ before $-\mathfrak{s}$. This is $\varphi_{2}(\mathfrak{s}, m)=$ $\mathfrak{s} /(\mathfrak{s}+m)$, as given in (4.6), and $\operatorname{Pr}_{x}[M=m]=\varphi_{2}(\mathfrak{s}, m)-\varphi_{2}(\mathfrak{s}, m+1)$. We find

$$
\operatorname{Pr}\left[D_{\mathbf{t}}=d\right]=\epsilon_{d} \sum_{m=d}^{\infty} \frac{r^{d}}{r^{m}} \frac{\mathfrak{s}}{(\mathfrak{s}+m)(\mathfrak{s}+m+1)} .
$$

We know from Corollary 4.13 that $C^{*}(\mathfrak{s}) \sim 2 \mathfrak{s} / G_{1}\left(o_{1}, o_{1}\right)$. Therefore (4.17) implies

$$
2 \mathfrak{s}^{2} q^{\mathfrak{s}} G(x, y) \sim G_{1}\left(o_{1}, o_{1}\right) G_{2}\left(o_{2}, o_{2}\right) \mathrm{E}_{x}\left(\frac{\mathfrak{s}^{2}}{\left(\mathfrak{s}+D_{\mathfrak{t}}\right) r^{D_{\mathfrak{t}}}}\right)-\operatorname{Rest}(\mathfrak{s}),
$$

where

$$
\operatorname{Rest}(\mathfrak{s})=\mathrm{E}_{x}\left(\frac{\mathfrak{s}^{2}}{\mathfrak{s}+D_{\mathbf{t}}} R^{*}\left(\mathfrak{s}, D_{\mathfrak{t}}\right)\right)
$$

Using (4.19), we can write

$$
\begin{aligned}
& \mathrm{E}_{x}\left(\frac{\mathfrak{s}^{2}}{\left(\mathfrak{s}+D_{\mathfrak{t}}\right) r^{D_{\mathfrak{t}}}}\right)=\sum_{d=0}^{\infty} f_{\mathfrak{s}}(d) \quad \text { with } \\
& f_{\mathfrak{s}}(d)=\operatorname{Pr}_{x}\left[D_{\mathfrak{t}}=d\right] \frac{\mathfrak{s}^{2}}{(\mathfrak{s}+d) r^{d}}=\epsilon_{d} \frac{\mathfrak{s}}{\mathfrak{s}+d} \sum_{m=d}^{\infty} r^{-m} \frac{\mathfrak{s}^{2}}{(\mathfrak{s}+m)(\mathfrak{s}+m+1)}
\end{aligned}
$$

Now $f_{\mathfrak{s}}(d)$ is increasing in $\mathfrak{s}$, and $f_{\mathfrak{s}}(d) \rightarrow f(d)$ with $f(0)=r /(r-1)$ and $f(d)=r^{-d}$ for $d \geqslant 1$. Monotone convergence implies that

$$
\sum_{d=0}^{\infty} f_{\mathfrak{s}}(d) \rightarrow \sum_{d=0}^{\infty} f(d)=\frac{r+1}{r-1}=B_{1}^{*}
$$

To conclude our asymptotic estimate, we have to show that the rest tends to zero. We expand

$$
\operatorname{Rest}(\mathfrak{s})=\sum_{d=0}^{\infty} \operatorname{Pr}_{x}\left[D_{\mathfrak{t}}=d\right] \frac{\mathfrak{s}^{2}}{\mathfrak{s}+d} R^{*}(\mathfrak{s}, d)=\sum_{d=0}^{\infty} f_{\mathfrak{s}}(d) r^{d} R^{*}(\mathfrak{s}, d) .
$$

We have $R^{*}\left(\mathfrak{s}, D_{\mathfrak{t}}\right)<G_{2}^{*}\left(Z_{\mathfrak{t}}^{2}, x_{2}\right)=r^{-D_{\mathbf{t}}} G_{2}\left(o_{2}, o_{2}\right)$ and $f_{\mathfrak{s}}(d) r^{d} R^{*}(\mathfrak{s}, d)<f(d) G_{2}\left(o_{2}, o_{2}\right)$. Also, $\sum_{d} f(d)<$ $\infty$. On the other hand, $\lim _{\mathfrak{s} \rightarrow \infty} f_{\mathfrak{s}}(d) r^{d} R^{*}(\mathfrak{s}, d)=0$ point-wise in $d$. Dominated convergence implies $\operatorname{Rest}(\mathfrak{s}) \rightarrow 0$.

We remark that in the proof we might have treated Case 1 in the same way as Case 2, by first determining the distribution of $D_{\mathfrak{t}}$ and then letting $\mathfrak{s} \rightarrow \infty$ (whence $\mathbf{t} \rightarrow \infty$ ). However, it is more likely that the method used above will lend itself to an extension to finite range (instead of nearest neighbour) random walks on $\mathrm{DL}(q, r)$ where $p(x, y)$ depends only on $\mathfrak{u}_{1}, \mathfrak{d}_{1}, \mathfrak{u}_{2}, \mathfrak{d}_{2}$.

Again, from the last proposition we can also deduce the asymptotics of $G(x, y)$ in the dual situation (I) of Fig. 4 by considering $P^{*}$. In this case we have $G^{*}(x, y)=G(x, y)$, since $\mathfrak{h}\left(x_{1}\right)-\mathfrak{h}\left(y_{1}\right)=0$. Thus, we only have to exchange $r \leftrightarrow q$.

4.20. Corollary. Referring to situation (I) of Fig. 4, if $x=x_{1} x_{2}$ and $y=y_{1} y_{2}$ with $\mathfrak{s}=\mathfrak{s}(x, y)$ are such that $\mathfrak{u}_{1}=\mathfrak{d}_{1}=0\left(y_{1}=x_{1}\right)$ and $\mathfrak{u}_{2}=\mathfrak{d}_{2}=\mathfrak{s} \rightarrow \infty$ then

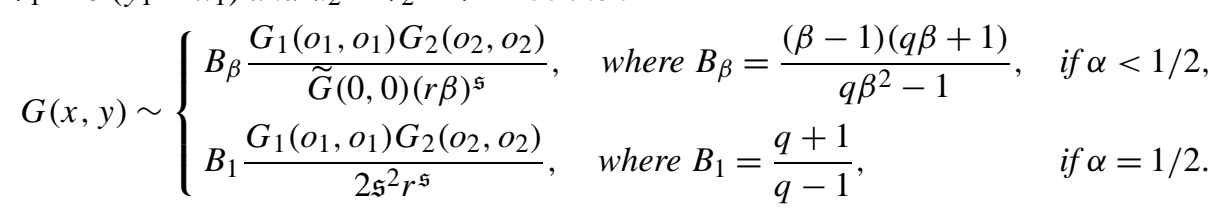


Combining Corollary 4.15, Proposition 4.16 and Corollary 4.20 with Proposition 4.9, we obtain Theorem 4.2.

\section{The Martin compactification}

We are now ready to determine the full Martin compactification of $P=P_{\alpha}$ on $\mathrm{DL}(q, r)$. Recall that the Martin compactification of the projected random walk $P_{\alpha, q}$ is $\widehat{\mathbb{T}}_{q}$, the end compactification of the tree. (The analogous result holds of course for the second projection $P_{1-\alpha, r}$ on $\mathbb{T}_{r}$.) The end compactification of $\mathbb{T}_{q}$ was described in $\S 2$; in particular, it is a compact metric space with the ultra-metric

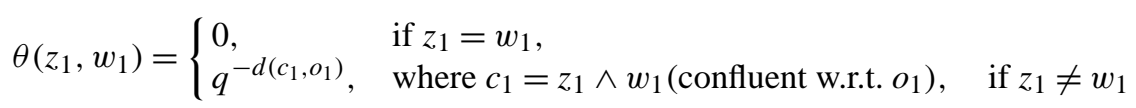

for $z_{1}, w_{1} \in \widehat{\mathbb{T}}_{q}$. In particular (recall), $z_{1}^{(n)} \rightarrow \xi_{1} \in \partial \mathbb{T}_{q}$ if and only if $d\left(z_{1}^{(n)} \wedge \xi_{1}, o_{1}\right) \rightarrow \infty$.

Since $\operatorname{DL}(q, r) \subset \mathbb{T}_{q} \times \mathbb{T}_{r}$, this provides us with a natural geometric compactification $\widehat{\operatorname{DL}}(q, r)$, namely, the closure of $\mathrm{DL}(q, r)$ in $\widehat{\mathbb{T}}_{q} \times \widehat{\mathbb{T}}_{r}$. The ideal boundary of $\mathrm{DL}$ in this compactification consists of 5 disjoint pieces:

$$
\left(\partial^{*} \mathbb{T}_{q} \times\left\{\omega_{2}\right\}\right) \cup\left(\left\{\omega_{1}\right\} \times \partial^{*} \mathbb{T}_{r}\right) \cup\left\{\omega_{1} \omega_{2}\right\} \cup\left(\mathbb{T}_{q} \times\left\{\omega_{2}\right\}\right) \cup\left(\left\{\omega_{1}\right\} \times \mathbb{T}_{r}\right),
$$

compare with [3]. For a sequence $y^{(n)}=y_{1}^{(n)} y_{2}^{(n)} \in \mathrm{DL}$, we have

$$
\begin{array}{llll}
y^{(n)} \rightarrow \xi_{1} \omega_{2}, & \xi_{1} \in \partial^{*} \mathbb{T}_{q}, & \text { if } y_{1}^{(n)} \rightarrow \xi_{1} & \text { and } \quad y_{2}^{(n)} \rightarrow \omega_{2} ; \\
y^{(n)} \rightarrow \omega_{1} \xi_{2}, & \xi_{2} \in \partial^{*} \mathbb{T}_{r}, & \text { if } y_{1}^{(n)} \rightarrow \omega_{1} & \text { and } \quad y_{2}^{(n)} \rightarrow \xi_{2} ; \\
y^{(n)} \rightarrow \omega_{1} \omega_{2}, & & \text { if } y_{1}^{(n)} \rightarrow \omega_{1} & \text { and } \quad y_{2}^{(n)} \rightarrow \omega_{2} ; \\
y^{(n)} \rightarrow y_{1} \omega_{2}, & y_{1} \in \mathbb{T}_{q}, & \text { if } y_{1}^{(n)}=y_{1} \forall n \geqslant n_{0} & \text { and } \quad y_{2}^{(n)} \rightarrow \omega_{2} ; \\
y^{(n)} \rightarrow \omega_{1} y_{2}, & y_{2} \in \mathbb{T}_{r}, & \text { if } y_{1}^{(n)} \rightarrow \omega_{1} & \text { and } \quad y_{2}^{(n)}=y_{2} \forall n \geqslant n_{0} .
\end{array}
$$

Every sequence in $\mathrm{DL}$ that tends to infinity has a subsequence of one of these 5 types.

Recall from $\S 3$ the Martin kernels associated with $P_{\alpha, q}$ and $P_{1-\alpha, r}$ and the spherical functions (3.7).

5.4. Theorem. If $\alpha=1 / 2$ then the Martin compactification of $\mathrm{DL}(q, r)$ with respect to $P=P_{1 / 2}$ is the geometric compactification $\widehat{\mathrm{DL}}(q, r)$. The extension of the Martin kernel on the boundary described in (5.2) and (5.3) is given by

(i) $K\left(x_{1} x_{2}, \xi_{1} \omega_{2}\right)=K_{1}\left(x_{1}, \xi_{1}\right), \quad \xi_{1} \in \partial^{*} \mathbb{T}_{q}$,

(ii) $K\left(x_{1} x_{2}, \omega_{1} \xi_{2}\right)=K_{2}\left(x_{2}, \xi_{2}\right), \quad \xi_{2} \in \partial^{*} \mathbb{T}_{r}$,

(iii) $K\left(x_{1} x_{2}, \omega_{1} \omega_{2}\right)=1$,

(iv) $K\left(x_{1} x_{2}, y_{1} \omega_{2}\right)=\frac{\phi_{\frac{1}{2}, q}\left(x_{1}, y_{1}\right)}{\phi_{\frac{1}{2}, q}\left(o_{1}, y_{1}\right)}, \quad y_{1} \in \mathbb{T}_{q}, \quad$ and

(v) $K\left(x_{1} x_{2}, \omega_{1} y_{2}\right)=\frac{\phi_{\frac{1}{2}, r}\left(x_{2}, y_{2}\right)}{\phi_{\frac{1}{2}, r}\left(o_{2}, y_{2}\right)}, \quad y_{2} \in \mathbb{T}_{r}$.

Each of the kernels in (i)-(iii) constitutes a minimal harmonic function, while the ones of (iv) and (v) are nonminimal harmonic. 
Proof. We use part (b) of Theorem 4.2.

(i) Suppose first that $y=y_{1} y_{2} \rightarrow \xi_{1} \omega_{2}$. Then, with the usual notation $\mathfrak{u}_{i}=\mathfrak{u}\left(x_{i}, y_{i}\right)$ and $\mathfrak{d}_{i}=\mathfrak{d}\left(x_{i}, y_{i}\right)$, we see that $\mathfrak{u}_{1}=d\left(x_{1}, x_{1} \wedge \xi_{1}\right)$ is constant when $y_{1}$ is sufficiently close to $\xi_{1}$. On the other hand, $\mathfrak{d}_{1}, \mathfrak{u}_{2}-\mathfrak{d}_{2} \rightarrow \infty$. Recall that $\mathfrak{u}_{1}+\mathfrak{u}_{2}=\mathfrak{d}_{1}+\mathfrak{d}_{2}=\mathfrak{s} \rightarrow \infty$. The dominant term in

$$
\frac{q+1}{q-1} \mathfrak{u}_{2} \mathfrak{d}_{2}+\mathfrak{s} \mathfrak{u}_{2} \mathfrak{d}_{1}+\mathfrak{s u}_{1} \mathfrak{d}_{2}+\frac{r+1}{r-1} \mathfrak{u}_{1} \mathfrak{d}_{1}
$$

is $\mathfrak{s u}_{2} \mathfrak{d}_{1}$, since

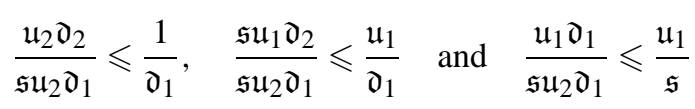

all tend to zero. As $\mathfrak{u}_{2} \sim \mathfrak{s}$, we find

$$
G(x, y) \sim A_{1} \frac{\mathfrak{d}_{1}}{\mathfrak{s}^{2} q^{\mathfrak{d}_{1}} r^{\mathfrak{d}_{2}}}=A_{1} \frac{\mathfrak{d}\left(x_{1}, y_{1}\right)}{\mathfrak{s}(x, y)^{2}} F_{1}\left(x_{1}, y_{1}\right) F_{2}\left(x_{2}, y_{2}\right) .
$$

For each $x \in \mathrm{DL}$ we have that $\mathfrak{s}(x, y)-\mathfrak{s}(o, y)$ and $\mathfrak{d}\left(x_{1}, y_{1}\right)-\mathfrak{d}\left(o_{1}, y_{1}\right)$ are constant when $y_{1}$ is close to $\xi_{1}$ in the end metric $\theta$. Therefore

$$
K(x, y) \sim \frac{\mathfrak{s}(o, y)^{2} \mathfrak{d}\left(x_{1}, y_{1}\right)}{\mathfrak{s}(x, y)^{2} \mathfrak{d}\left(o_{1}, y_{1}\right)} K_{1}\left(x_{1}, y_{1}\right) K_{2}\left(x_{2}, y_{2}\right) \rightarrow K_{1}\left(x_{1}, \xi_{1}\right) K_{2}\left(x_{2}, \omega_{2}\right)=K_{1}\left(x_{1}, \xi_{1}\right),
$$

since $K_{2}\left(\cdot, \omega_{2}\right) \equiv 1$.

(ii) follows immediately from (i), exchanging $r \leftrightarrow q$.

(iii) If $y=y_{1} y_{2} \rightarrow \omega_{1} \omega_{2}$ then $\mathfrak{u}_{i}=\mathfrak{u}\left(x_{i}, y_{i}\right) \rightarrow \infty$ for $i=1,2$. For given $x$, when $\mathfrak{u}_{i}>\mathfrak{u}\left(x_{i}, o_{i}\right)$ for $i=$ 1,2 , then $\mathfrak{d}_{i}=\mathfrak{d}\left(x_{i}, y_{i}\right)$ coincides with $\mathfrak{d}\left(o_{i}, y_{i}\right)$ and $\mathfrak{s}=\mathfrak{s}(x, y)$ coincides with $\mathfrak{s}(o, y)$, and we also have $\mathfrak{u}_{1}-$ $\mathfrak{u}\left(o_{1}, y_{1}\right)=\mathfrak{u}\left(o_{2}, y_{2}\right)-\mathfrak{u}_{2}=k$, where $k=\mathfrak{h}\left(x_{1}\right)$. Therefore

$$
K(x, y) \sim \frac{((q+1) /(q-1)) \mathfrak{u}_{2} \mathfrak{d}_{2}+\mathfrak{s u}_{2} \mathfrak{d}_{1}+\mathfrak{s u} \mathfrak{d}_{1} \mathfrak{d}_{2}+((r+1) /(r-1)) \mathfrak{u}_{1} \mathfrak{d}_{1}}{((q+1) /(q-1))\left(\mathfrak{u}_{2}+k\right) \mathfrak{d}_{2}+\mathfrak{s}\left(\mathfrak{u}_{2}+k\right) \mathfrak{d}_{1}+\mathfrak{s}\left(\mathfrak{u}_{1}-k\right) \mathfrak{d}_{2}+((r+1) /(r-1))\left(\mathfrak{u}_{1}-k\right) \mathfrak{d}_{1}} \rightarrow 1 .
$$

(iv) If $y_{1}$ remains fixed and $y_{2} \rightarrow \omega_{2}$, then $\mathfrak{d}_{1}=\mathfrak{d}\left(x_{1}, y_{1}\right)$ and $\mathfrak{u}_{1}=\mathfrak{u}\left(x_{1}, y_{1}\right)$ are constant. Since $\mathfrak{u}_{2}=\mathfrak{s}-\mathfrak{u}_{1}$ and $\mathfrak{d}_{2}=\mathfrak{s}-\mathfrak{d}_{1}$, where $\mathfrak{s}=\mathfrak{s}\left(x_{1}, y_{1}\right)$, we get

$$
\begin{aligned}
G(x, y) & \sim \frac{A_{1}}{\mathfrak{s}^{2} q^{\mathfrak{d}_{1}} r^{\mathfrak{d}_{2}}}\left(\frac{q+1}{q-1}\left(1-\frac{\mathfrak{u}_{1}}{\mathfrak{s}}\right)\left(1-\frac{\mathfrak{d}_{1}}{\mathfrak{s}}\right)+\left(1-\frac{\mathfrak{u}_{1}}{\mathfrak{s}}\right) \mathfrak{d}_{1}+\mathfrak{u}_{1}\left(1-\frac{\mathfrak{d}_{1}}{\mathfrak{s}}\right)+\frac{r+1}{r-1} \frac{\mathfrak{u}_{1} \mathfrak{d}_{1}}{\mathfrak{s}^{2}}\right) \\
& \sim \frac{A_{1}}{\mathfrak{s}^{2} q^{\mathfrak{d}_{1}} r^{\mathfrak{d}_{2}}}\left(\frac{q+1}{q-1}+\mathfrak{d}_{1}+\mathfrak{u}_{1}\right)=\frac{A_{1}}{\mathfrak{s}^{2} r^{\mathfrak{d}_{2}}} \phi_{\frac{1}{2}, q}\left(x_{1}, y_{1}\right) .
\end{aligned}
$$

As above in (iii), $\mathfrak{d}\left(o_{2}, y_{2}\right)=\mathfrak{d}_{2}\left(=\mathfrak{d}\left(x_{2}, y_{2}\right)\right)$ when $\mathfrak{u}\left(x_{2}, y_{2}\right)>\mathfrak{u}\left(x_{2}, o_{2}\right)$, and then also $\mathfrak{s}\left(o_{1}, y_{1}\right)-\mathfrak{s}=k$, a constant. Therefore,

$$
G(o, y) \sim \frac{A_{1}}{(\mathfrak{s}+k)^{2} r^{\mathfrak{d}_{2}}} \phi_{\frac{1}{2}, q}\left(o_{1}, y_{1}\right) .
$$

Thus, we obtain the proposed limit of $K(x, y)$ as $\left.y \rightarrow y_{1} \omega_{2}\right)$.

(v) follows from (iv), exchanging $r \leftrightarrow q$.

Finally, the - simple - proof of minimality of the functions in (i), (ii) and (iii) can be found in [28]. Nonminimality of the spherical functions in (iv) and (v) is straightforward, since they are also non-minimal for the projected random walks on the respective trees.

Next, we explain what happens in the case $\alpha \neq 1 / 2$. If $\left(y_{1}^{(n)}\right)$ is a sequence in $\mathbb{T}_{q}$ with $\mathfrak{u}\left(o_{1}, y_{1}^{(n)}\right) \rightarrow \infty$ then $y_{1}^{(n)} \rightarrow \omega_{1}$, independently of the values of $\mathfrak{h}\left(y_{1}^{(n)}\right)$. The horocyclic drawing of $\mathbb{T}_{q}$ as in Fig. 1 suggests that one may use a finer distinction by introducing boundary points $\omega_{1}^{k}, k \in \overline{\mathbb{Z}}=\mathbb{Z} \cup\{ \pm \infty\}$, at infinity, one for each horocycle, 
one at the "level" $-\infty$, and one at the "level" $+\infty$ (thinking of $\partial^{*} \mathbb{T}_{q}$ as the horocycle at $+\infty$ ). We set $\mathfrak{h}\left(\omega_{1}^{k}\right)=$ $k \in \overline{\mathbb{Z}}$. The new boundary is $\partial^{*} \mathbb{T}_{q} \cup\left\{\omega_{1}^{k}: k \in \overline{\mathbb{Z}}\right\}$. We write $\widetilde{\mathbb{T}}_{q}$ for the new compactification, which we call the horocyclic compactification. It is induced by the metric

$$
\theta_{h}\left(z_{1}, w_{1}\right)=\theta\left(z_{1}, w_{1}\right)+\left|\frac{\mathfrak{h}\left(z_{1}\right)}{1+\left|\mathfrak{h}\left(z_{1}\right)\right|}-\frac{\mathfrak{h}\left(w_{1}\right)}{1+\left|\mathfrak{h}\left(w_{1}\right)\right|}\right|,
$$

where $\theta$ is as in (5.1) and we set $\pm \infty /(1+\infty)= \pm 1$. In this metric, a sequence $\left(y_{1}^{(n)}\right)$ tends to $\xi_{1} \in \partial^{*} \mathbb{T}_{q}$ if and only if it converges to $\xi_{1}$ in the end topology. It tends to $\omega_{1}^{k}$ if and only if $\mathfrak{h}\left(y_{1}^{(n)}\right) \rightarrow k(k \in \overline{\mathbb{Z}})$ and $y_{1}^{(n)} \rightarrow \omega_{1}$ in the end topology.

Again, we can take the closure $\widetilde{\operatorname{DL}}(q, r)$ of $\mathrm{DL}(q, r)$ in $\widetilde{\mathbb{T}}_{q} \times \widetilde{\mathbb{T}}_{r}$, the horocyclic compactification of DL. In this case, the boundary consists of the following 5 disjoint pieces:

$$
\begin{gathered}
\left(\partial^{*} \mathbb{T}_{q} \times\left\{\omega_{2}^{-\infty}\right\}\right) \cup\left(\left\{\omega_{1}^{-\infty}\right\} \times \partial^{*} \mathbb{T}_{r}\right) \cup\left\{\omega_{1}^{k} \omega_{2}^{-k}: k \in \overline{\mathbb{Z}}\right\} \\
\cup\left\{y_{1} \omega_{2}^{-\mathfrak{h}\left(y_{1}\right)}: y_{1} \in \mathbb{T}_{q}\right\} \cup\left\{\omega_{1}^{-\mathfrak{h}\left(y_{2}\right)} y_{2}: y_{2} \in \mathbb{T}_{r}\right\} .
\end{gathered}
$$

We omit the detailed description of convergence, which is a straightforward adaptation of (5.3). The mapping $\omega_{i}^{k} \mapsto \omega_{i}(i=1,2)$ extends to a continuous surjection from the horocyclic onto the geometric compactification, which restricted to $\operatorname{DL}(q, r)$ is the identity.

5.7. Theorem. If $\alpha \neq 1 / 2$ then the Martin compactification of $\operatorname{DL}(q, r)$ with respect to $P=P_{\alpha}$ is the horocyclic compactification $\widetilde{\mathrm{DL}}(q, r)$. The extension of the Martin kernel on the boundary described in (5.6) is given by

(i) $K\left(x_{1} x_{2}, \xi_{1} \omega_{2}^{-\infty}\right)=K_{1}\left(x_{1}, \xi_{1}\right), \quad \xi_{1} \in \partial^{*} \mathbb{T}_{q}$,

(ii) $K\left(x_{1} x_{2}, \omega_{1}^{-\infty} \xi_{2}\right)=K_{2}\left(x_{2}, \xi_{2}\right), \quad \xi_{2} \in \partial^{*} \mathbb{T}_{r}$,

(iii) $K\left(x_{1} x_{2}, \omega_{1}^{k} \omega_{2}^{-k}\right)=\frac{\beta^{k}+\beta^{\mathfrak{h}\left(x_{1}\right)}}{\beta^{k}+1}, \quad k \in \overline{\mathbb{Z}}$,

(iv) $K\left(x_{1} x_{2}, y_{1} \omega_{2}^{-\mathfrak{h}\left(y_{1}\right)}\right)=\frac{\phi_{\alpha, q}\left(x_{1}, y_{1}\right)}{\phi_{\alpha, q}\left(o_{1}, y_{1}\right)}, \quad y_{1} \in \mathbb{T}_{q}, \quad$ and

(v) $K\left(x_{1} x_{2}, \omega_{1}^{-\mathfrak{h}\left(y_{2}\right)} y_{2}\right)=\frac{\phi_{1-\alpha, r}\left(x_{2}, y_{2}\right)}{\phi_{1-\alpha, r}\left(o_{2}, y_{2}\right)}, \quad y_{2} \in \mathbb{T}_{r}$.

In (iii), $\beta=(1-\alpha) / \alpha$, and for $k= \pm \infty$, the right-hand side is to be understood as the respective limit.

Each of the kernels in (i) and (ii) constitutes a minimal harmonic function, while the ones of (iii), (iv) and (v) are non-minimal harmonic.

Proof. Once more, the proof that the minimal harmonic functions are precisely those in (i) and (ii) can be found in $[28]$.

We now study convergence of $K(x, y)$ as $y$ tends to a boundary point. This time, we use part (a) of Theorem 4.2. We assume that $\alpha<1 / 2$, since the case $\alpha>1 / 2$ follows by exchanging $q \leftrightarrow r$ and using $\alpha^{*}=1-\alpha$ in the place of $\alpha$, or also by using the relation (4.12).

(i) Suppose that $y=y_{1} y_{2} \rightarrow \xi_{1} \omega_{2}^{-\infty}$ in $\widetilde{\mathrm{DL}}$. Then $y_{1} \rightarrow \xi_{1}$ and $y_{2} \rightarrow \omega_{2}$ in the end compactifications of the respective trees. We proceed as in the proof of Theorem 5.4 and find that in the formula of Theorem 4.2(a), the dominant one among the four terms in the (...) on the right-hand side is the second one. It behaves like $\beta^{-\mathfrak{d}_{2}}$. Therefore, using (3.5),

$$
G(x, y) \sim \frac{A_{\beta}}{(q \beta)^{\mathfrak{d}_{1}}(r \beta)^{\mathfrak{d}_{2}}}=A_{\beta} F_{1}\left(x_{1}, y_{1}\right) F_{2}^{*}\left(x_{2}, y_{2}\right),
$$


where (recall) $F_{2}^{*}\left(x_{2}, y_{2}\right)$ corresponds to exchanging $\alpha \leftrightarrow 1-\alpha$, that is, to the projection $P_{\alpha, r}$ onto $\mathbb{T}_{r}$ of $P_{\alpha}^{*}=$ $P_{1-\alpha}$. Therefore we obtain

$$
K(o, y) \sim K_{1}\left(x_{1}, y_{1}\right) K_{2}^{*}\left(x_{2}, y_{2}\right) \rightarrow K_{1}\left(x_{1}, \xi_{1}\right) K_{2}^{*}\left(x_{2}, \omega_{2}\right) .
$$

Noting that $K_{2}^{*}\left(x_{2}, \omega_{2}\right)=1$, we get the proposed Martin kernel.

(ii) Similarly, if $y=y_{1} y_{2} \rightarrow \omega_{1}^{\infty} \xi_{2}$ then the dominant term in the formula of Theorem 4.2(a) is the third one, which behaves like $\beta^{-\mathfrak{u}_{2}}$. This and (3.5) yield

$$
G(x, y) \sim \frac{A_{\beta}}{(q \beta)^{\mathfrak{d}_{1}} r^{\mathfrak{d}_{2}} \beta^{\mathfrak{u}_{2}}}=A_{\beta} F_{1}\left(x_{1}, y_{1}\right) F_{2}\left(x_{2}, y_{2}\right),
$$

this time without passing to $P^{*}$. The conclusion is now as in (i) above.

(iii) Let $y \rightarrow \omega_{1}^{k} \omega_{2}^{-k}$, so that $\mathfrak{u}_{i} \rightarrow \infty(i=1,2)$.

(a) $k=+\infty$. Then $\mathfrak{d}_{1}-\mathfrak{u}_{1}=\mathfrak{u}_{2}-\mathfrak{d}_{2} \rightarrow \infty$, so that the dominant term in the formula of Theorem 4.2(a) is the second one, as in (i). We get the same estimate as in (i), but have to replace $\xi_{1}$ with $\omega_{1}$, i.e.,

$$
K(x, y) \rightarrow K_{1}\left(x_{1}, \omega_{1}\right) K_{2}^{*}\left(x_{2}, \omega_{2}\right)=1 .
$$

(b) $k=-\infty$. In this case, the dominant term and asymptotic estimate of $G(x, y)$ are the same as in (ii), whence

$$
K(o, y) \rightarrow K_{1}\left(x_{1}, \omega_{1}\right) K_{2}\left(x_{2}, \omega_{2}\right)=\beta^{-\mathfrak{h}\left(x_{2}\right)}=\beta^{\mathfrak{h}\left(x_{1}\right)},
$$

since $K_{1}\left(x_{1}, \omega_{1}\right)=1$.

(c) $k \in \mathbb{Z}$, and $\mathfrak{h}\left(y_{1}\right)=k$. In this case, all of $\mathfrak{u}_{i}, \mathfrak{d}_{i}(i=1,2)$ tend to $\infty$. Also $\mathfrak{d}_{1}-\mathfrak{u}_{1}=\mathfrak{u}_{2}-\mathfrak{d}_{2}=k-\mathfrak{h}\left(x_{1}\right)$. Therefore, in the formula of Theorem 4.2(a), among the four terms in the (...) the second and the third one are of the same order and dominate the other two. We obtain

$$
G(x, y) \sim \frac{A_{\beta}}{(q \beta)^{\mathfrak{d}_{1}} r^{\mathfrak{d}_{2}}}\left(\frac{1}{\beta^{\mathfrak{d}_{2}}}+\frac{1}{\beta^{\mathfrak{u}_{2}}}\right)=A_{\beta} F_{1}\left(x_{1}, y_{1}\right) F_{2}^{*}\left(x_{2}, y_{2}\right)\left(1+\beta^{\mathfrak{h}\left(x_{1}\right)-k}\right) .
$$

Therefore

$$
K(x, y) \rightarrow K_{1}\left(x_{1}, \omega_{1}\right) K_{2}^{*}\left(x_{2}, \omega_{2}\right) \frac{1+\beta^{\mathfrak{h}\left(x_{1}\right)-k}}{1+\beta^{-k}}=\frac{\beta^{k}+\beta^{\mathfrak{h}\left(x_{1}\right)}}{\beta^{k}+1} .
$$

(iv) Recall that when $y_{1}$ remains fixed and $y_{2} \rightarrow \omega_{2}^{-\mathfrak{h}\left(y_{1}\right)}$, then $\mathfrak{d}_{1}=\mathfrak{d}\left(x_{1}, y_{1}\right)$ and $\mathfrak{u}_{1}=\mathfrak{u}\left(x_{1}, y_{1}\right)$ are constant, while $\mathfrak{u}_{2}=\mathfrak{s}-\mathfrak{u}_{1}$ and $\mathfrak{d}_{2}=\mathfrak{s}-\mathfrak{d}_{1}$. In Theorem 4.2(a), the first three of the four terms in the (..) are of the same order and dominate fourth. Thus

$$
G(x, y) \sim \frac{A_{\beta}}{(q \beta)^{\mathfrak{d}_{1}} r^{\mathfrak{d}_{2}}}\left(\frac{B_{\beta}}{\beta^{\mathfrak{s}}}+\frac{\beta^{\mathfrak{d}_{1}}-1}{\beta^{\mathfrak{s}}}+\frac{\beta^{\mathfrak{u}_{1}}-1}{\beta^{\mathfrak{s}}}\right)=A_{\beta}(\beta-1) F_{2}^{*}\left(x_{2}, y_{2}\right) \phi_{\alpha, q}\left(x_{1}, y_{1}\right) .
$$

This yields the proposed limit of $K(x, y)$.

(v) When $y_{2}$ is fixed and $y_{1} \rightarrow \omega_{1}^{-\mathfrak{h}\left(y_{2}\right)}$, we get analogously

$$
G(x, y) \sim \frac{A_{\beta}}{(q \beta)^{\mathfrak{d}_{1}} r^{\mathfrak{d}_{2}}}\left(\frac{B_{\beta}}{\beta^{\mathfrak{s}}}+\frac{\beta^{\mathfrak{d}_{1}}-1}{\beta^{\mathfrak{s}}}+\frac{\beta^{\mathfrak{u}_{1}}-1}{\beta^{\mathfrak{s}}}\right)=A_{\beta}(\beta-1) F_{1}^{*}\left(x_{1}, y_{1}\right) \phi_{1-\alpha, r}\left(x_{2}, y_{2}\right) .
$$

Again, this yields the proposed limit of $K(x, y)$.

\section{Positive eigenfunctions}

It is well known and easy to prove that positive $t$-harmonic functions $h$ (satisfying $P h=t \cdot h$ ) exist if and only if $t \geqslant \rho(P)$, see e.g. [27], Lemma 7.2. The Green kernel (resolvent) associated with eigenvalue $t$ is

$$
G(x, y \mid t)=\sum_{n=0}^{\infty} p^{(n)}(x, y) / t^{n}, \quad x, y \in X
$$


(Instead of the variable $t$, often $z=1 / t$ is used in the literature.) The Martin compactification associated with $P$ and the eigenvalue $t$ can be constructed in the same way as described in the Introduction, using the Martin kernel

$$
K(x, y \mid t)=G(x, y \mid t) / G(o, y \mid t) .
$$

Now consider $P=P_{\alpha}$ on $\operatorname{DL}(q, r)$, its projections to the two trees, and in particular, $\widetilde{P}$ on $\mathbb{Z}$. We fix $t \geqslant \rho=$ $\rho(P)=2 \sqrt{\alpha(1-\alpha)}$. Set

$$
\alpha(t)=\frac{t-\sqrt{t^{2}-\rho^{2}}}{2 t} \text { and } \lambda(t)=\frac{t-\sqrt{t^{2}-\rho^{2}}}{2 \alpha} .
$$

Then the function on $\mathbb{Z}$ defined by $\psi(k)=\lambda(t)^{k}$ satisfies $\widetilde{P}_{\alpha} \psi=t \cdot \psi$. We can lift this function to $\mathbb{T}^{1}, \mathbb{T}^{2}$ and $\mathrm{DL}$ by using the respective projection, and we obtain a $t$-harmonic function for the respective random walk. Then we can conjugate the respective transition matrix with the lifted function, and divide by $t$. We end up with a new transition matrix. On DL, this becomes

$$
\frac{p_{\alpha}(x, y) \psi\left(\mathfrak{h}\left(y_{1}\right)\right)}{t \psi\left(\mathfrak{h}\left(x_{1}\right)\right)}=p_{\alpha(t)}(x, y) .
$$

Consequently, the associated Green and Martin kernels on DL satisfy

$$
\begin{aligned}
& G_{\alpha}(x, y \mid t)=G_{\alpha(t)}(x, y) \lambda(t)^{\mathfrak{h}\left(x_{1}\right)-\mathfrak{h}\left(y_{1}\right)} \quad \text { and } \\
& K_{\alpha}(x, y \mid t)=\frac{G_{\alpha}(x, y \mid t)}{G_{\alpha}(o, y \mid t)}=K_{\alpha(t)}(x, y) \lambda(t)^{\mathfrak{h}\left(x_{1}\right)},
\end{aligned}
$$

where $G_{\alpha(t)}(x, y)$ and $K_{\alpha(t)}(x, y)$ are the ordinary Green and Martin kernels (with $\left.t=1\right)$ of $P_{\alpha(t)}$ on DL. Thus, the estimates of $\S 4$ also yield the asymptotics of $G_{\alpha}(x, y \mid t)$. Note here that $\alpha(\rho)=1 / 2$ and $\alpha(t)<1 / 2$ when $t>\rho$. Also note that formulas analogous to (6.1) and (6.2) hold for the projected random walks on the two trees.

6.3. Corollary. The Martin compactification of $\mathrm{DL}(q, r)$ with respect to $P_{\alpha}$ and eigenvalue $t$ is the geometric compactification $\widehat{\mathrm{DL}}(q, r)$ when $t=\rho\left(P_{\alpha}\right)$ and the horocyclic compactification $\widetilde{\mathrm{DL}}(q, r)$ when $t>\rho\left(P_{\alpha}\right)$.

We omit transcribing from $\S 5$ the explicit formulas for all the extended Martin kernels and just remark that for any $t \geqslant \rho$, we get

$$
\begin{array}{lll}
K\left(x_{1} x_{2}, \xi_{1} \omega_{2}^{-\infty} \mid t\right)=K_{1}\left(x_{1}, \xi_{1} \mid t\right), & \xi_{1} \in \partial^{*} \mathbb{T}_{q}, & \text { and } \\
K\left(x_{1} x_{2}, \omega_{1}^{-\infty} \xi_{2} \mid t\right)=K_{2}\left(x_{1}, \xi_{1} \mid t\right), & \xi_{2} \in \partial^{*} \mathbb{T}_{r} . &
\end{array}
$$

We have omitted the $\alpha$, resp. $1-\alpha$ in the subscripts, and the superscript of $\omega_{2}^{\infty}(i=1,2)$ has to be omitted when $t=\rho$.

Once more, the Martin compactification is stable in the sense of Picardello and Woess [21]: in particular, the compactification is the same for all $t>\rho$, while at the bottom of the positive spectrum, i.e., for $t=\rho$, it is smaller. Indeed, the identity on $\operatorname{DL}(q, r)$ extends to a continuous surjection from the horocyclic onto the geometric compactification.

\section{A remark on the elliptic Harnack inequality}

The elliptic Harnack inequality for reversible random walks on graphs appears frequently in recent research, see e.g. Hebisch and Saloff-Coste [15], Delmotte [6], Grigor'yan and Telcs [14], or - most suitable in our context - the recent note of Barlow [1]. Barlow shows among other that the elliptic Harnack inequality for a random walk 
with "controlled weights" (in particular, for SRW on a graph $X$ with bounded vertex degrees) is equivalent with a Harnack inequality for restricted Green functions

$$
G^{D}(x, y)=\sum_{n=0}^{\infty} \operatorname{Pr}\left[Z_{n}=y, Z_{k} \in D(k \leqslant n) \mid Z_{0}=x\right],
$$

where $D \subset X$ is finite.

In the formulation of [1], Theorem 2, this inequality - denoted (HG) - requires that there is a constant $C$ such that if $x_{0}, x, y \in X$ are such that $d\left(x_{0}, x\right)=d\left(x_{0}, y\right)=R \geqslant 1$ and $v \in D$ for all $v$ with $d\left(x_{0}, v\right) \leqslant 2 R$, then

$$
G^{D}\left(x_{0}, y\right) \leqslant C \cdot G^{D}\left(x_{0}, x\right) .
$$

When the random walk is transient then we can let $D$ tend to $X$ (i.e., we use an increasing sequence $\left(D_{n}\right)$ of finite subsets whose union is $X$ ), and we see that (HG) implies

$$
G\left(x_{0}, y\right) \leqslant C \cdot G\left(x_{0}, x\right) \text { for all } x_{0}, x, y \in X \text { with } d\left(x_{0}, x\right)=d\left(x_{0}, y\right) .
$$

In [1], it is shown that the random walk on the lamplighter group which corresponds to SRW on $\operatorname{DL}(2,2)$ does not satisfy (HG), or equivalently, the elliptic Harnack inequality.

This can also be seen easily from our asymptotic estimate. Indeed, consider SRW on $\operatorname{DL}(q, q)$ and $R \geqslant 1$. We choose $x=x_{1} x_{2}$ such that $\mathfrak{h}\left(x_{1}\right)=d(o, x)=2 R$, so that the relative position of $x$ with respect to $o$ is that of (III) in Fig. 4. Also, we choose $y=y_{1} y_{2}$ such that $\mathfrak{h}\left(y_{1}\right)=0$ and $d(o, y)=2 R$, with relative position as in (I) of Fig. 4. Then, using Corollaries 4.15 and 4.20 with $q=r$ and $\beta=1$, we get

$$
G(o, x) \sim \frac{G_{1}\left(o_{1}, o_{1}\right) G_{2}\left(o_{2}, o_{2}\right)}{4 R q^{2 R}} \text { and } G(o, y) \sim \frac{q+1}{q-1} \frac{G_{1}\left(o_{1}, o_{1}\right) G_{2}\left(o_{2}, o_{2}\right)}{2 R^{2} q^{R}},
$$

as $R \rightarrow \infty$. Thus, $G(o, x) / G(o, y) \rightarrow 0$, and (7.2) does not hold.

\section{References}

[1] M. Barlow, Some remarks on the elliptic Harnack inequality, Bull. London Math. Soc. 37 (2005) 200-208.

[2] L. Bartholdi, W. Woess, Spectral computations on lamplighter groups and Diestel-Leader graphs , J. Fourier Analysis Appl. 11 (2005) 175-202.

[3] D. Bertacchi, Random walks on Diestel-Leader graphs, Abh. Math. Sem. Univ. Hamburg 71 (2001) 205-224.

[4] P. Cartier, Fonctions harmoniques sur un arbre, Symposia Math. 9 (1972) 203-270.

[5] D.I. Cartwright, V.A. Kaimanovich, W. Woess, Random walks on the affine group of local fields and of homogeneous trees, Ann. Inst Fourier (Grenoble) 44 (1994) 1243-1288.

[6] Th. Delmotte, Graphs between the elliptic and parabolic Harnack inequalities, Potential Anal. 16 (2002) 151-168.

[7] W. Dicks, Th. Schick, The spectral measure of certain elements of the complex group ring of a wreath product, Geom. Dedicata 93 (2002) 121-137.

[8] R. Diestel, I. Leader, A conjecture concerning a limit of non-Cayley graphs, J. Algebraic Combin. 14 (2001) 17-25.

[9] J.L. Doob, Discrete potential theory and boundaries, J. Math. Mech. 8 (1959) 433-458.

[10] E.B. Dynkin, Boundary theory of Markov processes (the discrete case), Russian Math. Surveys 24 (1969) 1-42.

[11] E.B. Dynkin, M.B. Malyutov, Random walks on groups with a finite number of generators, Soviet Math. Dokl. 2 (1961) $399-402$.

[12] A.G. Erschler, On the asymptotics of the rate of departure to infinity, Zap. Nauchn. Sem. S.-Petersburg. Otdel. Mat. Inst. Steklov. (POMI) 283 (2001) 251-257, 263 (in Russian).

[13] R.I. Grigorchuk, A. Żuk, The lamplighter group as a group generated by a 2-state automaton, and its spectrum, Geom. Dedicata 87 (2001) 209-244.

[14] A. Grigor'yan, A. Telcs, Harnack inequalities and sub-Gaussian estimates for random walks, Math. Ann. 324 (2002) $521-556$.

[15] W. Hebisch, L. Saloff-Coste, On the relation between elliptic and parabolic Harnack inequalities, Ann. Inst. Fourier (Grenoble) 51 (2001) 1437-1481.

[16] G.A. Hunt, Markoff chains and Martin boundaries, Illinois J. Math. 4 (1960) 313-340.

[17] V.A. Kaimanovich, Poisson boundaries of random walks on discrete solvable groups, in: H. Heyer (Ed.), Probability Measures on Groups X, Plenum, New York, 1991, pp. 205-238. 
[18] V.A. Kaimanovich, A.M. Vershik, Random walks on discrete groups: boundary and entropy, Ann. Probab. 11 (1983) 457-490.

[19] J.G. Kemeny, J.L. Snell, Finite Markov Chains, reprint of the 1960 edition, Springer, New York, 1976.

[20] R. Lyons, R. Pemantle, Y. Peres, Random walks on the lamplighter group, Ann. Probab. 24 (1996) 1993-2006.

[21] M.A. Picardello, W. Woess, Examples of stable Martin boundaries of Markov chains, in: M. Kishi (Ed.), Potential Theory, de Gruyter, Berlin, 1990, pp. 261-270.

[22] C. Pittet, L. Saloff-Coste, Amenable groups, isoperimetric profiles and random walks, in: Geometric Group Theory Down Under (Canberra, 1996), de Gruyter, Berlin, 1999, pp. 293-316.

[23] C. Pittet, L. Saloff-Coste, On random walks on wreath products, Ann. Probab. 30 (2002) 948-977.

[24] D. Revelle, Rate of escape of random walks on wreath products, Ann. Probab. 31 (2003) 1917-1934.

[25] D. Revelle, Heat kernel asymptotics on the lamplighter group, Electronic Comm. Probab. 8 (2003) 142-154.

[26] L. Saloff-Coste, W. Woess, Transition operators, groups, norms, and spectral radii, Pacific J. Math. 180 (1997) $333-367$.

[27] W. Woess, Random Walks on Infinite Graphs and Groups, Cambridge Tracts in Math., vol. 138, Cambridge University Press, Cambridge, 2000.

[28] W. Woess, Lamplighters, Diestel-Leader graphs, random walks, and harmonic functions, Combinatorics, Probability \& Computing 14 (2005) 415-433. 